\title{
Alkene-Alkyne Coupling as a Linchpin: An Efficient and Convergent Synthesis of Amphidinolide P
}

Barry M. Trost* and Julien P.N. Papillon

Department of Chemistry, Stanford University, Stanford, California 94305-5080

\section{Experimentals.}

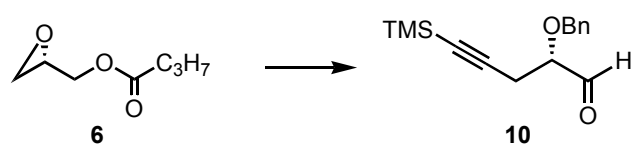

To a solution of trimethylsilylacetylene $(9.44 \mathrm{~g}, 96.14 \mathrm{mmol})$ in ether $(400 \mathrm{~mL})$ at $-78{ }^{\circ} \mathrm{C}$ was added $n \mathrm{BuLi}(2.58 \mathrm{M}$ in hexanes, $35.5 \mathrm{~mL}, 91.6 \mathrm{mmol}$ ) dropwise over $20 \mathrm{~min}$ and the mixture was stirred for another $25 \mathrm{~min}$, whereupon $\mathrm{AlMe}_{3}(2.0 \mathrm{M}$ in hexanes, $45.8 \mathrm{~mL}$, $91.6 \mathrm{~mL})$ was added dropwise over $25 \mathrm{~min}$. The mixture was warmed up to $-40{ }^{\circ} \mathrm{C}$ and stirred at this temperature for $35 \mathrm{~min}$, whereupon it was recooled to $-78{ }^{\circ} \mathrm{C}$. (S)-Glycidyl butyrate (6) $(10.15 \mathrm{~g}, 70.40 \mathrm{mmol})$ in ether $(70$ $\mathrm{mL})$ was added via cannula over $15 \mathrm{~min}$ and after $10 \mathrm{~min}, \mathrm{BF}_{3} \cdot \mathrm{Et}_{2} \mathrm{O}(12.99 \mathrm{~g}, 91.6 \mathrm{mmol})$ was added over $10 \mathrm{~min}$. The mixture was stirred at $-78^{\circ} \mathrm{C}$ for $25 \mathrm{~min}$, whereupon $0.5 \mathrm{M}$ aqueous $\mathrm{HCl}(200 \mathrm{~mL})$ was carefully added and the cooling bath was removed. Upon reaching r.t., 0.5M aqueous $\mathrm{HCl}(100$ $\mathrm{mL})$ and EtOAc $(800 \mathrm{~mL})$ were added. The organic phase was washed with saturated aqueous $\mathrm{NaHCO}_{3}$ and brine. The combined organic phase was dried over $\mathrm{MgSO}_{4}$ and the volatiles were removed in vacuo to give alcohol 7 (16.97 g) as a colorless oil. A portion (16.36 g) was redissolved in dry dioxane $(200 \mathrm{~mL})$. Freshly prepared crude benzyl trichloroacetimidate (33.9 g, $134 \mathrm{mmol})$ was added, followed with triflic acid $(1.96 \mathrm{~g}, 13.08 \mathrm{mmol})$. An exothermic reaction ensued and after 15 min, TLC analysis showed complete conversion. After 25 min, water $(100 \mathrm{~mL})$ and ether $(400 \mathrm{~mL})$ were added and the mixture was stirred for $15 \mathrm{~min}$. After further dilution with ether $(300 \mathrm{~mL})$, the combined organic phase was washed with saturated aqueous $\mathrm{NaHCO}_{3}$ and brine. The combined aqueous phase was back-extracted once with ether. After drying the combined organic phase over $\mathrm{MgSO}_{4}$, the volatiles were removed in vacuo to give a residue (22.5 g, quant.) which was used without further purification. An analytical sample was obtained by purification by silica gel flash chromatography (petroleum ether-ethyl acetate, 19:1) to afford the alkyne $\mathbf{8}$ as a colorless oil (Found: C, 68.82; H, 8.70. $\mathrm{C}_{19} \mathrm{H}_{28} \mathrm{O}_{3} \mathrm{Si}$ requires C, 68.63; H, 8.49\%); $[\alpha]_{\mathrm{D}}^{22}+10.9$ (c 0.68, $\mathrm{CHCl}_{3}$ );

$\mathrm{R}_{\mathrm{f}} 0.60$ (petroleum ether-ethyl acetate, 4:1); $v_{\max } / \mathrm{cm}^{-1} 2962,1740,1250,1176,1101,843,698 ; \delta_{\mathrm{H}}$ (400 MHz, $\left.\mathrm{CDCl}_{3}\right) 0.15$ (9 H, s), 0.95 (3 H, t, J 7.0), 1.66 (2 H, sex, J 7.0), 2.31 (2 H, t, J 7.0), 2.52 (1 H, dd, J 17.0, 7.0), 2.57 (1 H, dd, J 17.5, 6.0), 3.78 (1 H, m), 4.17 (1 H, dd, J 11.5, 5.5), 4.31 (1 H, dd, $J 11.5,4.0), 4.64$ (1 H, d, J 12.0), 4.68 (1 H, d, J 12.0), 7.28-7.42 (5 H, m); $\delta_{\mathrm{C}}\left(100 \mathrm{MHz}, \mathrm{CDCl}_{3}\right) 0.0$, 13.7, 18.4, 23.0, 36.1, 64.6, 71.9, 75.2, 87.0, 102.5, 127.6, 127.8, 128.4, 138.0, 173.4. 
To a mixture of crude $8(23.4 \mathrm{~g}, 70.4 \mathrm{mmol})$ in $\mathrm{CH}_{2} \mathrm{Cl}_{2}(300 \mathrm{~mL})$ at $-78{ }^{\circ} \mathrm{C}$ was added DIBAL-H $(1.0 \mathrm{M}$ in hexane, $93 \mathrm{~mL}, 93 \mathrm{mmol})$ over $30 \mathrm{~min}$. The mixture was stirred at $-78{ }^{\circ} \mathrm{C}$ for another 15 min, whereupon a saturated solution of Rochelle salt $(300 \mathrm{~mL})$ was added. The mixture was allowed to warm up to r.t. and after $2 \mathrm{~h}$ of vigorous stirring, the two phases were separated and the aqueous phase was extracted three times with $\mathrm{CH}_{2} \mathrm{Cl}_{2}$. The combined organic phase was dried over $\mathrm{MgSO}_{4}$ and the volatiles were removed in vacuo to give the alcohol $9(18.5 \mathrm{~g})$ as a colorless oil. A portion (223 mg) was redissolved in $\mathrm{CH}_{2} \mathrm{Cl}_{2}(1 \mathrm{~mL})$. To a solution of DMSO $(265 \mathrm{mg}, 3.39 \mathrm{mmol})$ in $\mathrm{CH}_{2} \mathrm{Cl}_{2}(4 \mathrm{~mL})$ at $-78{ }^{\circ} \mathrm{C}$ was added oxalyl chloride $(215 \mathrm{mg}, 1.70 \mathrm{mmol})$ and the mixture was stirred for $20 \mathrm{~min}$, whereupon the solution of alcohol was added dropwise. After another $20 \mathrm{~min}$ at $-78{ }^{\circ} \mathrm{C}$, triethylamine $(0.43 \mathrm{~g}, 4.23 \mathrm{mmol})$ was added and the cooling bath was removed. Upon reaching 0 ${ }^{\circ} \mathrm{C}$, the mixture was partitioned between ether and saturated aqueous $\mathrm{NH}_{4} \mathrm{Cl}$. The organic phase was washed with saturated aqueous $\mathrm{NaHCO}_{3}$, brine, and dried over $\mathrm{MgSO}_{4}$. Concentration in vacuo and purification by flash silica gel column chromatography (petroleum ether-ethyl acetate, 9:1 to 4:1) afforded aldehyde $10(163 \mathrm{mg}, 0.63 \mathrm{mmol}, 71 \%)$ as a yellow oil; $[\alpha]_{\mathrm{D}}^{22}+32.6\left(c 2.00, \mathrm{CHCl}_{3}\right)$; $\mathrm{R}_{\mathrm{f}} 0.14$ (petroleum ether-ethyl acetate, 9:1); $v_{\max } / \mathrm{cm}^{-1} 2959,2342,2179,1738,1455,1250,1112$, 1030, 845, 760, 698; $\delta_{\mathrm{H}}\left(300 \mathrm{MHz}, \mathrm{CDCl}_{3}\right) 0.17(9 \mathrm{H}, \mathrm{s}), 2.63(1 \mathrm{H}, \mathrm{dd}, J 17.5,7.0), 2.72(1 \mathrm{H}, \mathrm{dd}, J$ 17.5, 6.0), 3.94 (1 H, dt, J 7.0, 6.0), $4.75(2 \mathrm{H}, \mathrm{s}), 7.28-7.42(5 \mathrm{H}, \mathrm{m}), 9.68(1 \mathrm{H}, \mathrm{br} . \mathrm{s}) ; \delta_{\mathrm{C}}(100 \mathrm{MHz}$, $\left.\mathrm{CDCl}_{3}\right)-0.1,21.8,72.7,80.8,87.8,100.9,128.0,128.1,128.5,137.0,201.2$.

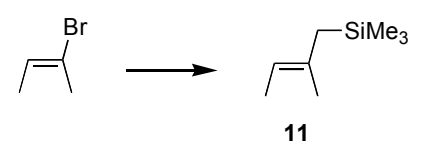

To a mixture of 2-bromo-2-butene (Aldrich, 7:3 mixture of isomers, $5.04 \mathrm{~g}, 37.37 \mathrm{mmol}$ ) and $\mathrm{Ni}(\mathrm{acac})_{2}(0.58 \mathrm{~g}, 2.25 \mathrm{mmol})$ in ether $(20 \mathrm{~mL})$ at $0{ }^{\circ} \mathrm{C}$ was added trimethylsilylmagnesium chloride $(1.0 \mathrm{M}$ in ether, $49 \mathrm{~mL}, 49 \mathrm{mmol})$ over $15 \mathrm{~min}$. The mixture was warmed to r.t. and stirred for another $21 \mathrm{~h}$, whereupon saturated aqueous $\mathrm{NH}_{4} \mathrm{Cl}$ was added. The solids were dissolved by vigorous stirring for $10 \mathrm{~min}$ and the aqueous phase was extracted several times with pentane. The combined organic phase was dried over $\mathrm{MgSO}_{4}$, filtered and concentrated by distillation using a 10 cm Vigreux column. The residue was distilled with a Kugelrohr apparatus (oven temperature 170 $\left.{ }^{\circ} \mathrm{C}\right)$ to give the product as a colourless oil $(0.80 \mathrm{~g})$. The viscous brown residue was further distilled while applying a slight vacuum (oven temperature $170{ }^{\circ} \mathrm{C}$ ) to afford a pale yellow oil $(1.99 \mathrm{~g}$ ). Both fractions were of satisfactory purity as judged using ${ }^{1} \mathrm{H}$ NMR spectroscopy and were combined to give the title compound $11(2.79 \mathrm{~g}, 19.6 \mathrm{mmol}, 52 \%)$ as a pale yellow oil and a 1:1 $\mathrm{E} / \mathrm{Z}$ mixture; $\delta_{\mathrm{H}}$ $\left(300 \mathrm{MHz}, \mathrm{CDCl}_{3}\right) 0.00$ and $0.03(9 \mathrm{H}, \mathrm{s}), 1.46-1.67(8 \mathrm{H}, \mathrm{m}), 4.95-5.05$ and $5.06-5.14(1 \mathrm{H}, \mathrm{m})$. 
These data were consistent with those reported in the literature: Ishiara, K.; Mouri, M.; Gao, Q.; Maruyama, T.; Furuta, K.; Yamamoto, H. J. Am. Chem. Soc. 1993, 115, 11490.

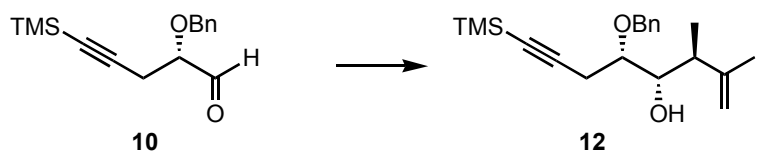

To a solution of $10(1.07 \mathrm{~g}, 4.10 \mathrm{mmol})$ in $\mathrm{CH}_{2} \mathrm{Cl}_{2}(12 \mathrm{~mL})$ and pentane $(11 \mathrm{~mL})$ at $-110{ }^{\circ} \mathrm{C}$ (ethanol- liquid $\mathrm{N}_{2}$ bath) was added $\mathrm{SnCl}_{4}\left(1.0 \mathrm{M}\right.$ in $\left.\mathrm{CH}_{2} \mathrm{Cl}_{2}, 4.1 \mathrm{~mL}, 4.1 \mathrm{mmol}\right)$ over $5 \mathrm{~min}$. The bath was removed and the dark orange mixture was stirred until it became homogeneous (ca. 3 min), whereupon it was lowered back into the cooling bath and stirred for 10 min. Silane 11 (1.17 g, $8.22 \mathrm{mmol}$ ) was added neat dropwise. After $15 \mathrm{~min}$, saturated aqueous $\mathrm{NaHCO}_{3}(5 \mathrm{~mL})$ was added and the cooling bath was removed. Upon reaching r.t. the mixture was diluted with ethyl acetate (50 $\mathrm{mL})$ and saturated aqueous $\mathrm{NaHCO}_{3}(25 \mathrm{~mL})$. The white precipitate that formed was filtered off through celite. The two phases were separated and the organic phase was washed with brine and dried over $\mathrm{MgSO}_{4}$. Concentration in vacuo and purification by flash silica gel column chromatography (petroleum ether-ethyl acetate, 19:1) afforded the alcohol 12 (1.04 g, 3.15 mmol, 77 \%) as a pale yellow oil and an inseparable 9:1 mixture of diastereomers (Found: C, 72.80; H, 9.37. $\mathrm{C}_{20} \mathrm{H}_{30} \mathrm{O}_{2} \mathrm{Si}$ requires C, 72.67; H, $9.15 \%$ ); $[\alpha]_{\mathrm{D}}^{22}+13.8$ (c $0.55, \mathrm{CHCl}_{3}$ ); $\mathrm{R}_{\mathrm{f}} 0.13$ (petroleum etherethyl acetate, 19:1); $v_{\max } / \mathrm{cm}^{-1} 3565,2962,2342,2176,1738,1542,1250,1063,1029,843,760,698$; $\delta_{\mathrm{H}}\left(500 \mathrm{MHz}, \mathrm{CDCl}_{3}\right.$, minor diastereomer in brackets) $0.15(0.14)(9 \mathrm{H}, \mathrm{s}), 0.91(1.16)(3 \mathrm{H}, \mathrm{d}, J$ 7.0), 1.71 (1.68) (3 H, s), 2.47 (1 H, dq, J 9.0, 7.0), 2.65 (1 H, dd, J 17.0, 7.0), 2.69 (1 H, dd, J 17.0, 6.0), 3.58 (1 H, dd, J 9.0, 2.0), 3.65 (1 H, ddd, J 7.0, 6.0, 2.0), 4.54 (4.50) (1 H, d, J 11.5), 4.80 (4.70) (1 H, d, J 11.5), 4.80-4.84 (4.72-4.77) (2 H, m), 7.30-7.37 (5 H, m); $\delta_{\mathrm{C}}\left(125 \mathrm{MHz}, \mathrm{CDCl}_{3}\right)$ 0.0, 15.7, 18.7, $22.3,44.0,72.3,73.5,76.5,86.8,104.1,112.6,127.9,128.1,128.4,137.9,147.7$.

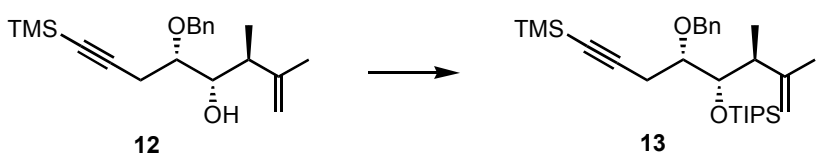

To a solution of $12(3.0 \mathrm{~g}, 9.07 \mathrm{mmol})$ in $\mathrm{CH}_{2} \mathrm{Cl}_{2}(50 \mathrm{~mL})$ at $0{ }^{\circ} \mathrm{C}$ was added 2,6-lutidine $(3.9 \mathrm{~g}$, $36.05 \mathrm{mmol})$ and TIPSOTf $(8.3 \mathrm{~g}, 27.16 \mathrm{mmol})$. The solution was warmed to $24{ }^{\circ} \mathrm{C}$ and stirred for $6 \mathrm{~h}$, whereupon it was diluted with ether and washed with saturated aqueous $\mathrm{NH}_{4} \mathrm{Cl}$, saturated aqueous $\mathrm{NaHCO}_{3}$ and brine. After drying over $\mathrm{MgSO}_{4}$, the mixture was concentrated in vacuo, and the residue was purified by flash silica gel column chromatography (petroleum ether-ethyl acetate, 1 to $3 \%)$ to give the alkyne $13(3.63 \mathrm{~g}, 7.45 \mathrm{mmol}, 82 \%)$ as a colorless oil; $[\alpha]_{\mathrm{D}}{ }^{23}+26.7$ (c 1.07 , 
$\mathrm{CHCl}_{3}$ ); $\mathrm{R}_{\mathrm{f}} 0.20$ (petroleum ether-ethyl acetate, 49:1); $v_{\max } / \mathrm{cm}^{-1} 2945,2867,1464,1250,1094,884$, 843, 679; $\delta_{\mathrm{H}}\left(400 \mathrm{MHz}, \mathrm{CDCl}_{3}\right) 0.15(9 \mathrm{H}, \mathrm{s}), 1.05(21 \mathrm{H}, \mathrm{s}), 1.17$ (3 H, d, J 7.0), 1.80 (3 H, s), 2.422.50 (1 H, m), 2.49 (1 H, dd, J 17.0, 5.0), 2.69 (1 H, dd, J 17.0, 5.0), 3.51 (1 H, q, J 5.0), 4.13 (1 H, t, J 5.0), 4.51 (1 H, d, J 12.0), 4.68 (1 H, d, J 12.0), 4.77 (1 H, br. s), 4.79 (1 H, br. s), 7.23-7.38 (5 H, $\mathrm{m}) ; \delta_{\mathrm{C}}\left(125 \mathrm{MHz}, \mathrm{CDCl}_{3}\right)$ 0.0, 13.1, 15.9, 18.3, 21.2, 21.8, 44.3, 71.4, 75.0, 79.5, 86.1, 104.6, 112.0, 127.2, 127.4, 128.0, 138.9, 147.9.

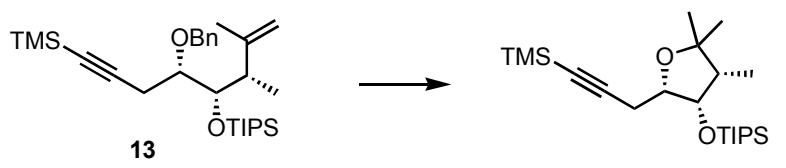

To a stirred solution of $13(19 \mathrm{mg}, 0.039 \mathrm{mmol})$ in $\mathrm{CH}_{2} \mathrm{Cl}_{2}(0.4 \mathrm{~mL})$ at $-78{ }^{\circ} \mathrm{C}$ was added 9-Br-9BBN (1.0 M in $\left.\mathrm{CH}_{2} \mathrm{Cl}_{2}, 0.05 \mathrm{~mL}, 0.05 \mathrm{mmol}\right)$ and the mixture was stirred for $5 \mathrm{~min}$, whereupon acetic acid $(15 \mu \mathrm{l})$ was added and the resulting red solution was warmed to $0{ }^{\circ} \mathrm{C} .1 \mathrm{M}$ Aqueous $\mathrm{Na}_{2} \mathrm{~S}_{2} \mathrm{O}_{3}$ was added, followed with brine and the mixture was extracted with ether. After drying over $\mathrm{MgSO}_{4}$, the mixture was concentrated in vacuo, and the residue was purified by flash silica gel column chromatography (petroleum ether-ether, 0 to $3 \%$ ) to give the tetrahydrofuran derivative (9 $\mathrm{mg}, 0.022 \mathrm{mmol}, 59 \%$ ) as a colorless oil; $[\alpha]_{\mathrm{D}}^{23}+29.3$ (c 1.39, $\mathrm{CHCl}_{3}$ ); $\mathrm{R}_{\mathrm{f}} 0.10$ (petroleum etherether, 9:1); $v_{\max } / \mathrm{cm}^{-1} 2962,2868,2168,1465,1249,1168,1140,1059,1014,843,680 ; \delta_{\mathrm{H}}(500 \mathrm{MHz}$, $\left.\mathrm{CDCl}_{3}\right) 0.13(9 \mathrm{H}, \mathrm{s}), 1.01(3 \mathrm{H}, \mathrm{d}, J$ 7.5), $1.13(21 \mathrm{H}, \mathrm{s}), 1.17(3 \mathrm{H}, \mathrm{s}), 1.20(3 \mathrm{H}, \mathrm{s}), 2.00$ (1 H, qd, 7.5, 4.5), 2.47 (1 H, dd, J 17.0, 5.5), 2.63 (1 H, dd, J 17.0, 8.0), 4.00 (1 H, ddd, J 8.0, 5.5, 4.0), 4.43 (1 $\mathrm{H}, \mathrm{dd}, J 4.5,4.0)$.

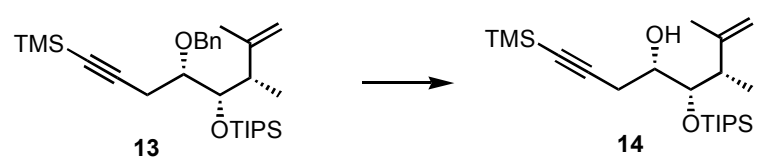

To a solution of 13 (3.63 g, $7.45 \mathrm{mmol})$ in a 9:1 mixture of dichloroethane/aqueous buffer 7 (50 $\mathrm{mL}$ ) was added DDQ (3.38 g, $14.89 \mathrm{mmol})$, and the solution was heated to reflux for $45 \mathrm{~min}$. After allowing the mixture to cool to r.t., it was partitionned between water and ether. The organic phase was washed three times with water and brine. The combined aqueous phase was back-extracted once with ether. After drying over $\mathrm{MgSO}_{4}$, the combined organic phase was concentrated in vacuo, and the residue was purified by flash silica gel column chromatography (petroleum ether-ether, 2 to 3\%) to give alcohol $14(2.43 \mathrm{~g}, 6.12 \mathrm{mmol}, 82 \%$ ) as a colorless oil (Found: C, 66.61; H, 11.36. $\mathrm{C}_{22} \mathrm{H}_{44} \mathrm{O}_{2} \mathrm{Si}_{2}$ requires $\mathrm{C}, 66.60 ; \mathrm{H}, 11.18 \%$ ); $[\alpha]_{\mathrm{D}}^{23}+3.5\left(c 0.72, \mathrm{CHCl}_{3}\right) ; \mathrm{R}_{\mathrm{f}} 0.32$ (petroleum ether- 
ether, 19:1); $v_{\max } / \mathrm{cm}^{-1} 3452,2945,2868,2175,1464,1250,1104,1053,1016,883,844,680,652 ; \delta_{\mathrm{H}}$ (500 MHz, C6 $\left.\mathrm{D}_{6}\right) 0.17$ (9 H, s), $1.09(21 \mathrm{H}, \mathrm{s}), 1.32$ (3 H, d, J 7.0), 1.87 (3 H, s), 2.43-2.50 (1 H, m), 2.51 (1 H, dd, J 16.5, 9.5), 2.59 (1 H, dd, J 16.5, 5.0), 2.75 (1 H, d, J 9.5), 3.94 (1 H, tdd, J 9.5, 5.0, 1.5), 4.44 (1 H, dd, J 3.5, 1.5), 4.87 (1 H, br. s), 4.95 (1 H, br. s); $\delta_{\mathrm{C}}\left(100 \mathrm{MHz} \mathrm{CDCl}_{3}\right) 0.0,12.3$, 12.9, 18.2, 23.2, 27.3, 46.0, 67.8, 71.7, 86.7, 103.6, 112.2, 145.8 .

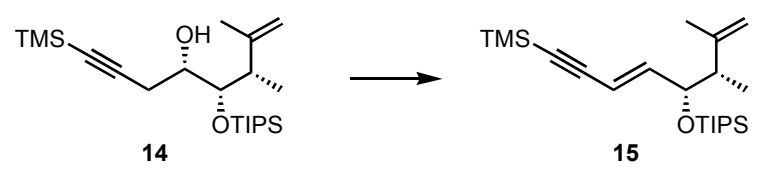

To a solution of $14(1.73 \mathrm{~g}, 4.36 \mathrm{mmol})$ and triphenylphosphine $(3.46 \mathrm{~g}, 13.19 \mathrm{mmol})$ in dry toluene $(20 \mathrm{~mL})$ was added diisopropyl azodicarboxylate $(2.67 \mathrm{~g}, 13.20 \mathrm{mmol})$ and the flask was lowered into a preheated oil bath $\left(80^{\circ} \mathrm{C}\right)$. After stirring at this temperature for $20 \mathrm{~min}$, the volatiles were removed in vacuo and the residue was purified by flash silica gel column chromatography (petroleum ether) to give alkyne $15(1.37 \mathrm{~g}, 3.61 \mathrm{mmol}, 83 \%)$ as a colorless oil and an 8:1 inseparable $E / Z$ mixture (Found: C, 69.59; H, 11.14. $\mathrm{C}_{22} \mathrm{H}_{42} \mathrm{OSi}_{2}$ requires C, 69.77; H, $11.18 \%$ ); $[\alpha]_{\mathrm{D}}^{23}+1.7(c 3.41$, $\mathrm{CHCl}_{3}$ ); $\mathrm{R}_{\mathrm{f}} 0.40$ (petroleum ether); $v_{\max } / \mathrm{cm}^{-1} 2945,2868,2361,2134,1464,1250,1059,958,883$, $843,760,679,654$

$\boldsymbol{E}$ isomer: $\delta_{\mathrm{H}}\left(500 \mathrm{MHz}, \mathrm{CDCl}_{3}\right) 0.18(9 \mathrm{H}, \mathrm{s}), 0.97(3 \mathrm{H}, \mathrm{d}, J$ 7.0), $1.07(21 \mathrm{H}, \mathrm{s}), 1.76(3 \mathrm{H}, \mathrm{s}), 2.40$ (1 H, br. quin., J 6.0), 4.46 (1 H, td, J 5.0, 2.0), 4.75 (1 H, s), 4.85 (1 H, s), 5.70 (1 H, dd, J 16.0, 2.0), 6.09 (1 H, dd, $J 16.0,5.0) ; \delta_{\mathrm{C}}\left(125 \mathrm{MHz}, \mathrm{CDCl}_{3}\right)$ 0.0, 12.3, 12.5, 18.1, 22.2, 47.1, 74.1, 94.1, 103.8, 110.0, 111.9, 144.5, 146.0.

$Z$ isomer: $\delta_{\mathrm{H}}\left(500 \mathrm{MHz}, \mathrm{CDCl}_{3}\right) 0.17(9 \mathrm{H}, \mathrm{s}), 0.97(3 \mathrm{H}, \mathrm{d}, J 7.0), 1.06(21 \mathrm{H}, \mathrm{s}), 1.80(3 \mathrm{H}, \mathrm{s}), 2.40$ (1 H, masked), 4.46 (1 H, masked), $4.75(1 \mathrm{H}, \mathrm{s}), 4.85(1 \mathrm{H}, \mathrm{s}), 5.49$ (1 H, d, J 11.5), 5.89 (1 H, dd, J $11.5,9.0)$.

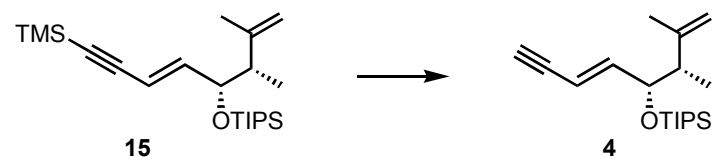

To a stirred solution of $15(1.60 \mathrm{~g}, 4.22 \mathrm{mmol})$ in methanol $(60 \mathrm{~mL})$ was added potassium carbonate $(0.59 \mathrm{~g}, 4.26 \mathrm{mmol})$ and the slurry was stirred for $2 \mathrm{~h}$ (a homogeneous solution was obtained after 1 h). Evaporation to dryness gave a residue that was partitioned between ether and saturated aqueous ammonium chloride. The organic phase was washed with water and brine. The aqueous phase was back-extracted once with ether. The combined organic phase was dried over $\mathrm{MgSO}_{4}$ and concentrated in vacuo to give alkyne $4(1.24 \mathrm{~g}, 4.04 \mathrm{mmol}, 96 \%)$ as a colorless oil and an 8:1 inseparable $E / Z$ mixture (Found: $M^{+}$, 306.2385. $\mathrm{C}_{19} \mathrm{H}_{34} \mathrm{OSi}$ requires $M$ 306.2379, 2.1 ppm, EIMS); 
$[\alpha]_{\mathrm{D}}^{23}+7.6\left(c 1.56, \mathrm{CHCl}_{3}\right) ; \mathrm{R}_{\mathrm{f}} 0.40$ (petroleum ether); $v_{\max } / \mathrm{cm}^{-1} 3315,2944,2868,1464,1374,1127$, 1061, 959, 883;

$\boldsymbol{E}$ isomer: $\delta_{\mathrm{H}}\left(400 \mathrm{MHz}, \mathrm{CDCl}_{3}\right) 0.98(3 \mathrm{H}, \mathrm{d}, J$ 7.2), $1.07(21 \mathrm{H}, \mathrm{s}), 1.77(3 \mathrm{H}, \mathrm{s}), 2.42$ (1 H, broad quin., J 6.5), 2.85 (1 H, d, J 2.5), 4.46 (1 H, td, J 5.0, 1.5), 4.74 (1 H, s), 4.85 (1 H, s), 5.66 (1 H, ddd, $J$ 16.0, 2.5, 1.5), 6.17 (1 H, dd, J 16.0, 5.0); $\delta_{\mathrm{C}}\left(100 \mathrm{MHz}, \mathrm{CDCl}_{3}\right)$ 12.3, 12.4, 18.0, 22.1, 47.0, 74.1, 77.1, 82.2, 108.9, 112.0, 144.5, 145.9.

$Z$ isomer: $\delta_{\mathrm{H}}\left(400 \mathrm{MHz}, \mathrm{CDCl}_{3}\right) 0.98(3 \mathrm{H}, \mathrm{d}, J$ 7.2), $1.06(21 \mathrm{H}, \mathrm{s}), 1.79(3 \mathrm{H}, \mathrm{s}), 2.42(1 \mathrm{H}$, masked), 2.85 (1 H, d, J 2.5), 4.46 (1 H, masked), $4.74(1 \mathrm{H}, \mathrm{s}), 4.85$ (1 H, s), 5.48 (1 H, dd, J 11.0, 2.5), 5.95 (1 H, dd, J11.0, 9.0).

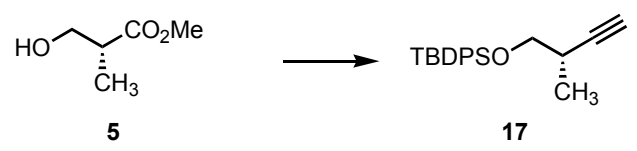

To a solution of (R)-hydroxyisobutyric acid methyl ester (5, $9.34 \mathrm{~g}, 79.06 \mathrm{mmol}$ ) and imidazole (7.06 g, $102.82 \mathrm{mmol})$ in $\mathrm{CH}_{2} \mathrm{Cl}_{2}(90 \mathrm{~mL})$ was added TBDPSCl $(21.7 \mathrm{~g}, 79.09 \mathrm{mmol})$ at $0{ }^{\circ} \mathrm{C}$. The mixture was warmed up to r.t. and stirred for $30 \mathrm{~min}$, whereupon saturated aqueous $\mathrm{NaHCO}_{3}(200$ $\mathrm{mL}$ ) was added. The mixture was vigorously stirred for one minute and extracted with $\mathrm{CH}_{2} \mathrm{Cl}_{2}$. The organic layer was washed with brine, dried and the volatiles were removed in vacuo to give the ester 16 (28.0 g, quant.) as a colorless oil; $\mathrm{R}_{\mathrm{f}} 0.35$ (petroleum ether-ethyl acetate, 9:1); $\delta_{\mathrm{H}}(300 \mathrm{MHz}$, $\left.\mathrm{CDCl}_{3}\right) 1.03(9 \mathrm{H}, \mathrm{s}), 1.16(3 \mathrm{H}, \mathrm{d}, J$ 7.0), 2.71 (1 H, sex, J 7.0), $3.68(3 \mathrm{H}, \mathrm{s}), 3.72(1 \mathrm{H}, \mathrm{dd}, J$ 9.5, 5.5), 3.82 (1 H, dd, J 9.5, 7.0), 7.35-7.43 (6 H, m), 7.64 (4 H, d, J 6.5). A portion (4.10 g) was redissolved in $\mathrm{CH}_{2} \mathrm{Cl}_{2}(60 \mathrm{~mL})$, cooled to $-78{ }^{\circ} \mathrm{C}$ and DIBAL-H $(1.0 \mathrm{M}$ in hexanes, $13.4 \mathrm{~mL}, 13.4$ $\mathrm{mmol}$ ) was added via syringe pump over $30 \mathrm{~min}$. After another $30 \mathrm{~min}$, methanol $(0.50 \mathrm{~g}, 15.6$ mmol) was added. The cooling bath was removed and the mixture was allowed to warm to r.t. A solution of (1-diazo-2-oxo-propyl)-phosphonic acid dimethyl ester (5.53 g, $28.78 \mathrm{mmol}$ ) in THF $(100 \mathrm{~mL})$ was cooled to $-78{ }^{\circ} \mathrm{C}$ and sodium methoxide (25\%wt in methanol, $\left.6.6 \mathrm{~mL}, 28.84 \mathrm{mmol}\right)$ in THF $(20 \mathrm{~mL})$ was added over $15 \mathrm{~min}$, followed by the aldehyde solution, which was cannulated over $10 \mathrm{~min}$. The cooling bath was removed, and after $20 \mathrm{~min}$, a saturated aqueous solution of Rochelle's salt $(200 \mathrm{~mL})$ was added. The mixture was vigorously stirred for $30 \mathrm{~min}$, and the two phases were separated. The aqueous phase was extracted twice with ether and the combined organic phase was dried over $\mathrm{MgSO}_{4}$. Evaporation of the solvent in vacuo gave a residue which was purified by silica gel flash chromatography (petroleum ether-ether, 2 to $4 \%$ ) to afford the alkyne 17 (3.07 g, $9.52 \mathrm{mmol}, 83 \%$ ) as a colorless oil (Found: $M^{+}, 322.1749 . \mathrm{C}_{21} \mathrm{H}_{26} \mathrm{OSi}$ requires $M$ 322.1753, $1.3 \mathrm{ppm}$, 
EIMS); $[\alpha]_{\mathrm{D}}{ }^{26}-5.3$ (c 4.10, $\mathrm{CHCl}_{3}$ ); $\mathrm{R}_{\mathrm{f}} 0.28$ (petroleum ether-ether, $2 \%$ ); $v_{\max } / \mathrm{cm}^{-1} 3309,2932$, 2859, 1472, 1428, 1389, 1112, 824, 740, 702; $\delta_{\mathrm{H}}\left(400 \mathrm{MHz}, \mathrm{CDCl}_{3}\right) 1.06(9 \mathrm{H}, \mathrm{s}), 1.23$ (3 H, d, J 7.0), 2.02 (1 H, d, J 2.5), 2.56 (1 H, sexd, J 7.0, 2.5), 3.54 (1 H, dd, J 9.5, 7.5), 3.73 (1 H, dd, J 9.5, 6.0), 7.35-7.43 (6 H, m), 7.67-7.70 (4 H, m); $\delta_{\mathrm{C}}\left(100 \mathrm{MHz} \mathrm{CDCl}_{3}\right)$ 17.3, 19.3, 26.8, 28.8, 67.4, 69.0, 86.5, 127.6, 129.6, 133.5, 135.6.

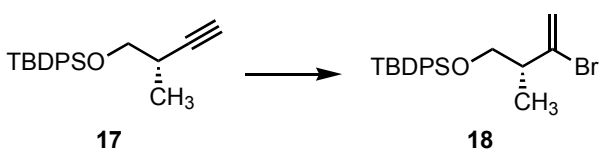

To a solution of alkyne $17(15.59 \mathrm{~g}, 48.34 \mathrm{mmol})$ in $\mathrm{CH}_{2} \mathrm{Cl}_{2}(150 \mathrm{~mL})$ and hexane $(150 \mathrm{~mL})$ at -20 ${ }^{\circ} \mathrm{C}$ was added 9-Br-9-BBN (unopened commercial batch, $1.0 \mathrm{M}$ in $\mathrm{CH}_{2} \mathrm{Cl}_{2}, 100 \mathrm{~mL}, 100 \mathrm{mmol}$ ) over $10 \mathrm{~min}$. The mixture was stirred for a further $6 \mathrm{~h}$ at $0{ }^{\circ} \mathrm{C}$ and acetic acid was added $(39.9 \mathrm{~g}, 664$ mmol). After a further $1 \mathrm{~h}$ at $0{ }^{\circ} \mathrm{C}, 1 \mathrm{M}$ aqueous $\mathrm{Na}_{2} \mathrm{~S}_{2} \mathrm{O}_{3}(400 \mathrm{~mL})$ was added, followed with brine (400 mL). The milky aqueous phase was extracted with ether until it was clear $(3 \times 300 \mathrm{~mL})$. The combined organic phase was dried over $\mathrm{MgSO}_{4}$ and evaporation of the solvent in vacuo gave a residue that was purified by silica gel flash chromatography (petroleum ether- ether, 1 to $2 \%$ ) to afford the bromide 18 (18.76 g, $46.50 \mathrm{mmol}, 96 \%$ ) as a colorless oil (Found: C, 62.38; H, 6.82. $\mathrm{C}_{21} \mathrm{H}_{27} \mathrm{BrOSi}$ requires C, 62.52; $\mathrm{H}, 6.75 \%$ ); $[\alpha]_{\mathrm{D}}{ }^{26}-5.3$ (c 3.6, $\mathrm{CHCl}_{3}$ ); $\mathrm{R}_{\mathrm{f}} 0.40$ (petroleum etherether, $2 \%) ; v_{\max } / \mathrm{cm}^{-1} 2931,2858,2360,2342,1428,1112,823,739,701 ; \delta_{\mathrm{H}}\left(400 \mathrm{MHz}, \mathrm{CDCl}_{3}\right) 1.05$ (9 H, s), 1.08 (3 H, d, J 7.0), 2.60 (1 H, sex, J 7.0), 3.55 (1 H, dd, J 10.0, 6.0), 3.69 (1 H, dd, J 10.0, 7.0), 5.48 (1 H, d, J 1.5), 5.67 (1 H, dd, J 1.5, 0.5), 7.36-7.44 (6 H, m), 7.66 (2 H, dd, J 3.0, 1.5), 7.68 $(2 \mathrm{H}, \mathrm{dd}, J 3.0,1.0) ; \delta_{\mathrm{C}}\left(100 \mathrm{MHz}, \mathrm{CDCl}_{3}\right) 16.2$, 19.3, 26.8, 46.7, 66.3, 117.0, 127.6, 129.6, 133.6, 133.7, $135.6(2), 137.9$.

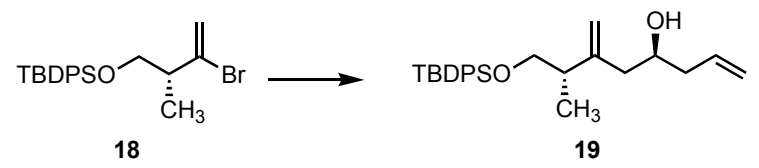

To a solution of thiophene $(0.76 \mathrm{~g}, 9.03 \mathrm{mmol})$ in THF $(8 \mathrm{~mL})$ at $-30{ }^{\circ} \mathrm{C}$ was added $n-\mathrm{BuLi}(2.58 \mathrm{M}$, $3.50 \mathrm{~mL}, 9.03 \mathrm{mmol}$ ) dropwise. The mixture was stirred for $30 \mathrm{~min}$, whereupon it was cannulated into a slurry of $\mathrm{CuCN}(99.99 \%, 809 \mathrm{mg}, 9.03 \mathrm{mmol})$ in $\mathrm{THF}(8 \mathrm{~mL})$ at $-78{ }^{\circ} \mathrm{C}$. The cooling bath was removed and upon reaching r.t., a clear brown solution was obtained. This solution was kept at ca. $-20{ }^{\circ} \mathrm{C}$ until the the vinyl lithium reagent was ready (vide infra).

To a solution of vinyl bromide $18(2.79 \mathrm{~g}, 6.91 \mathrm{mmol})$ in ether $(28 \mathrm{~mL})$ was added $t$-BuLi $(1.44 \mathrm{M}$, $10 \mathrm{~mL}, 14.4 \mathrm{mmol})$ at $-78{ }^{\circ} \mathrm{C}$ over $10 \mathrm{~min}$. After another $45 \mathrm{~min}$, the freshly prepared solution of 2 - 
thienyl lithiumcyanocuprate was cannulated into it. The pale brown heterogeneous mixture was warmed up to $-45^{\circ} \mathrm{C}$ (chlorobenzene/dry ice bath), and stirred at this temperature for $1 \mathrm{~h}$. A solution of (R)-glycidyl tosylate (TCI America, $3.1 \mathrm{~g}, 13.58 \mathrm{mmol}$ ) in THF (11 $\mathrm{mL})$ was then cannulated into the mixture, and the the resulting slurry was warmed up to $0^{\circ} \mathrm{C}$ over $10 \mathrm{~min}$. After an additional $5 \mathrm{~h}$ at $0{ }^{\circ} \mathrm{C}$, the mixture was recooled to $-78{ }^{\circ} \mathrm{C}$ and a vinyl lithium solution (13.93 mmol, prepared from $n \mathrm{BuLi}$ and tetravinyltin at $-78^{\circ} \mathrm{C}, 45 \mathrm{~min}$ then warming to $\left.24^{\circ} \mathrm{C}\right)$ in $\mathrm{THF}(14$ $\mathrm{mL})$ was added, followed after $5 \mathrm{~min}$, with $\mathrm{BF}_{3} \cdot \mathrm{Et}_{2} \mathrm{O}(1.97 \mathrm{~g}, 13.93 \mathrm{mmol})$. The resulting mixture was stirred for $20 \mathrm{~min}$, then quenched with a 9:1 solution of saturated aqueous $\mathrm{NH}_{4} \mathrm{Cl}$ solution/ $\mathrm{NH}_{4} \mathrm{OH}$ and diluted with ether. After 20 min of vigorous stirring followed by filtration through Celite, the organic phase was washed with brine. The combined aqueous phase was backextracted twice with ether. After drying the combined organic phase over $\mathrm{MgSO}_{4}$, the volatiles were removed in vacuo to give a residue that was purified by silica gel flash chromatography (petroleum ether-ethyl acetate, 19:1 to 9:1) to afford the alcohol 19 (2.01 g, $4.92 \mathrm{mmol}, 71 \%)$ as a colorless oil (Found: C, 76.43; H, 9.02. $\mathrm{C}_{26} \mathrm{H}_{36} \mathrm{O}_{2} \mathrm{Si}$ requires C, 76.42; H, $8.88 \%$ ); $[\alpha]_{\mathrm{D}}^{22}-13.1$ (c 3.22, $\mathrm{CHCl}_{3}$ ); $\mathrm{R}_{\mathrm{f}}$ 0.30 (petroleum ether-ethyl acetate, 9:1); $v_{\max } / \mathrm{cm}^{-1}$ 3448, 2960, 2931, 2858, 1472, 1428, 1121, 1080 , 823, 740, 702, 614; $\delta_{\mathrm{H}}\left(400 \mathrm{MHz}, \mathrm{CDCl}_{3}\right) 1.05$ (9 H, s), 1.07 (3 H, d, J 7.0), $2.04(1 \mathrm{H}, \mathrm{dd}, J$ 14.0, 9.5), 2.19-2.23 (3 H, m), 2.35 (1 H, broad sex, J 7.0), 3.49 (1 H, dd, J 10.0, 7.0), 3.62 (1 H, dd, J 10.0, 6.0), 3.71 (1 H, dddd, J 9.5, 6.0, 6.0, 4.5), 4.93 (1 H, s), $4.94(1 \mathrm{H}, \mathrm{s}), 5.09-5.14(2 \mathrm{H}, \mathrm{m}), 5.83(1 \mathrm{H}$, ddt, J 17.0, 10.5, 7.0), 7.35-7.43 (6 H, m), 7.64-7.68 (4 H, m); $\delta_{\mathrm{C}}\left(100 \mathrm{MHz}, \mathrm{CDCl}_{3}\right)$ 16.7, 19.2, 26.8, 41.4, 43.6, 68.2, 68.5, 112.7, 117.5, 127.6, 129.6, 133.6, 133.7, 134.9, 135.6, 135.6, 148.8.

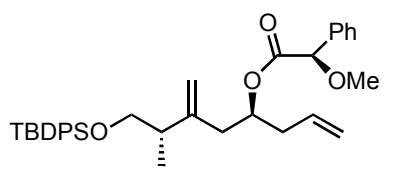

A dry tube was charged with alcohol $19(7 \mathrm{mg}, 0.017 \mathrm{mmol})$, (R)-methoxyphenylacetic acid (6 mg, $0.036 \mathrm{mmol})$ and DMAP $(0.2 \mathrm{mg}, 0.002 \mathrm{mmol}) \cdot \mathrm{CH}_{2} \mathrm{Cl}_{2}(0.2 \mathrm{~mL})$ was added, and after cooling to 0 ${ }^{\circ} \mathrm{C}$, DCC $(7.0 \mathrm{mg}, 0.034 \mathrm{mmol})$ was added, followed by warming to r.t. After $20 \mathrm{~min}$, the solvent was blown off with argon, the residue was taken up in petroleum ether and placed on a plug of silica gel. Elution with petroleum ether, then with $5 \%$ petroleum ether-ethyl acetate gave the mandelate ester (9 mg, $0.016 \mathrm{mmol}, 95 \%$ ) as a colorless oil; $\mathrm{R}_{\mathrm{f}} 0.41$ (petroleum ether-ethyl acetate, 9:1); $\delta_{\mathrm{H}}\left(500 \mathrm{MHz}, \mathrm{CDCl}_{3}\right) 1.03(9 \mathrm{H}, \mathrm{s}), 1.04$ (3 H, d, J 6.5), 2.05-2.18 (2 H, m), 2.15 (1 H, dd, J 14.5, 5.0), 2.22 (1 H, dd, J 14.5, 9.0), 2.27-2.34 (1 H, m), 3.38 (3 H, s), 3.43 (1 H, dd, J 9.5, 6.5), 3.58 
(1 H, dd, J 9.5, 5.5), 4.65 (1 H, s), 4.74-4.81 (4 H, m), 5.07 (1 H, ddt, J 10.0, 7.0, 4.5), 5.39 (1 H, ddt, J 17.5, 10.0, 7.0), 7.29-7.44 (11 H, m), 7.63-7.67 (4 H, m).

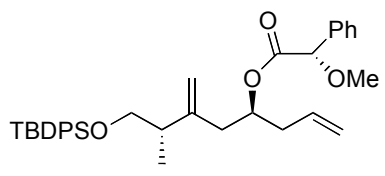

A dry tube was charged with alcohol $19(7 \mathrm{mg}, 0.017 \mathrm{mmol}),(S)$-methoxyphenylacetic acid (6 $\mathrm{mg}$, $0.036 \mathrm{mmol})$ and DMAP (0.2 $\mathrm{mg}, 0.002 \mathrm{mmol}) \cdot \mathrm{CH}_{2} \mathrm{Cl}_{2}(0.2 \mathrm{~mL})$ was added, and after cooling to 0 ${ }^{\circ} \mathrm{C}$ DCC $(7.0 \mathrm{mg}, 0.034 \mathrm{mmol})$ was added, followed by warming to r.t. After $20 \mathrm{~min}$, the solvent was blown off with argon, the residue was taken up in petroleum ether and placed on a plug of silica gel. Elution with petroleum ether, then with $5 \%$ petroleum ether-ethyl acetate gave the the mandelate ester ( $9 \mathrm{mg}, 0.016 \mathrm{mmol}, 95 \%$ ) as a colorless oil; $\mathrm{R}_{\mathrm{f}} 0.41$ (petroleum ether-ethyl acetate, 9:1); $\delta_{\mathrm{H}}\left(500 \mathrm{MHz}, \mathrm{CDCl}_{3}\right) 0.88(3 \mathrm{H}, \mathrm{d}, J 7.0), 1.01(9 \mathrm{H}, \mathrm{s}), 2.05-2.30(5 \mathrm{H}, \mathrm{m}), 3.32(1 \mathrm{H}, \mathrm{dd}, J$ 10.0, 7.0), 3.38 (3 H, s), 3.46 (1 H, dd, J 10.0, 5.5), 4.43 (1 H, d, J 1.0), 4.47 (1 H, s), 4.66 (1 H, s), 4.99-5.09 (3 H, m), 5.66 (1 H, ddt, J 17.5, 10.5, 7.5), 7.25-7.30 (3 H, m), 7.35-7.43 (8 H, m), 7.61$7.66(4 \mathrm{H}, \mathrm{m})$.

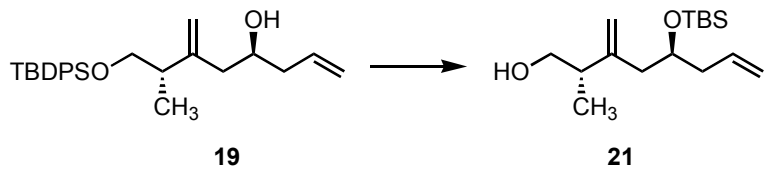

To a solution of 19 (3.08 g, $7.53 \mathrm{mmol})$ and 2,6-lutidine $(3.22 \mathrm{~g}, 30.05 \mathrm{mmol})$ in $\mathrm{CH}_{2} \mathrm{Cl}_{2}(40 \mathrm{~mL})$ was added TBSOTf $(3.57 \mathrm{~g}, 13.49 \mathrm{mmol})$ at $0{ }^{\circ} \mathrm{C}$. The mixture was stirred for $5 \mathrm{~min}$, diluted with ether and washed with saturated aqueous $\mathrm{NH}_{4} \mathrm{Cl}$ and brine. After drying the combined organic phase over $\mathrm{MgSO}_{4}$, the volatiles were removed in vacuo to give a residue which was redissolved in anhydrous DMF $(150 \mathrm{~mL})$. Acetic acid (0.51 g, $8.55 \mathrm{mmol})$ was added, followed with solid TBAF. $3 \mathrm{H}_{2} \mathrm{O}(2.66 \mathrm{~g}, 8.43 \mathrm{mmol})$. The solution was stirred for $13 \mathrm{~h}$, whereupon it was diluted with ether, washed with saturated $\mathrm{NH}_{4} \mathrm{Cl}$, saturated aqueous $\mathrm{NaHCO}_{3}$ and brine and dried over $\mathrm{MgSO}_{4}$. The volatiles were removed in vacuo to give a residue that was purified by silica gel flash chromatography (petroleum ether-ether, 9:1 to 4:1) to afford the alcohol 21 (1.65 g, $5.80 \mathrm{mmol}, 77$ \%) as a colorless oil (Found: $\left[M H-{ }^{t} B u\right]^{+}, 227.1457 . \mathrm{C}_{12} \mathrm{H}_{23} \mathrm{O}_{2} \mathrm{Si}$ requires $M H{ }^{-} \mathrm{B} u$ 227.1467, $4.5 \mathrm{ppm}$, EIMS); $[\alpha]_{\mathrm{D}}^{22}-8.4$ (c $\left.0.81, \mathrm{CHCl}_{3}\right) ; \mathrm{R}_{\mathrm{f}} 0.16$ (petroleum ether-ethyl acetate, 9:1); $\mathrm{v}_{\max } / \mathrm{cm}^{-1} 3355$, 2957, 2929, 2857, 1472, 1255, 1085, 836, 774; $\delta_{\mathrm{H}}\left(500 \mathrm{MHz}, \mathrm{CDCl}_{3}\right) 0.04$ (3 H, s), 0.06 (3 H, s), 0.88 (9 H, s), 1.06 (3 H, d, J 7.0), 2.17-2.23 (2 H, m), 2.25-2.31 (2 H, m), 2.37 (1 H, broad sex, J 6.0), 3.51 (1 H, dd, J 11.0, 5.5), 3.55 (1 H, dd, J 11.0, 6.5), 3.88 (1 H, quin, J 7.0), 4.92 (1 H, t J 1.0), 4.94 (1 H, 
td, $J 1.5,1.0), 5.02-5.06$ (2 H, m), 5.82 (1 H, ddt, J 15.0, 11.0, 6.5); $\delta_{\mathrm{C}}\left(100 \mathrm{MHz}, \mathrm{CDCl}_{3}\right)-4.4,16.1$, 18.1, 25.9, 41.6, 41.9, 42.3, 66.0, 71.0, 112.6, 117.1, 135.0, 147.9.

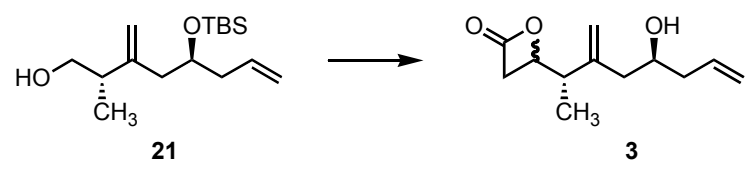

To a solution of DMSO $(1.68 \mathrm{~g}, 21.56 \mathrm{mmol})$ in $\mathrm{CH}_{2} \mathrm{Cl}_{2}(75 \mathrm{~mL})$ at $-78{ }^{\circ} \mathrm{C}$ was added oxalyl chloride $(1.36 \mathrm{~g}, 10.77 \mathrm{mmol})$ and the mixture was stirred for $20 \mathrm{~min}$, whereupon a solution of alcohol 21 (1.54 g, $5.41 \mathrm{mmol}$ ) was added dropwise. After another $20 \mathrm{~min}$ at $-78{ }^{\circ} \mathrm{C}$, triethylamine (3.26 g, $32.29 \mathrm{mmol}$ ) was added and the cooling bath was removed. Upon reaching $0{ }^{\circ} \mathrm{C}$, the mixture was partitioned between ether and saturated aqueous $\mathrm{NH}_{4} \mathrm{Cl}$. The organic phase was washed with saturated aqueous $\mathrm{NH}_{4} \mathrm{Cl}$, brine, dried over $\mathrm{MgSO}_{4}$ and concentrated in vacuo. The crude aldehyde, which was obtained as a yellow oil $(1.55 \mathrm{~g})$, was immediately redissolved in $\mathrm{CH}_{2} \mathrm{Cl}_{2}$ $(50 \mathrm{~mL})$ and cooled to $-78{ }^{\circ} \mathrm{C}$. $\mathrm{Me}_{2} \mathrm{AlCl}(1.0 \mathrm{M}$ in hexanes, $5.4 \mathrm{~mL}, 5.4 \mathrm{mmol})$ was added over $5 \mathrm{~min}$. The bright yellow mixture was stirred for $3 \mathrm{~min}$, whereupon neat trimethylsilylketene $(0.65 \mathrm{~g}, 5.72$ mmol) was added dropwise. After another $30 \mathrm{~min}, 0.5 \mathrm{M}$ aqueous $\mathrm{NaHSO}_{4}(20 \mathrm{~mL})$ and ether $(100$ $\mathrm{mL}$ ) were added and the mixture was allowed to warm to r.t. with vigorous stirring. Additional $0.5 \mathrm{M}$ aqueous $\mathrm{NaHSO}_{4}(150 \mathrm{~mL})$ and ether $(100 \mathrm{~mL})$ were added and the two clear phases were separated. The organic phase was washed with brine $(100 \mathrm{~mL})$ and the combined organic phase was back-extracted with ether $(2 \times 50 \mathrm{~mL})$, dried over $\mathrm{MgSO}_{4}$ and concentrated in vacuo. The yellow residue $(2.15 \mathrm{~g})$ was taken up in acetonitrile $(60 \mathrm{~mL})$ and $\mathrm{KF} \cdot 2 \mathrm{H}_{2} \mathrm{O}(0.76 \mathrm{~g}, 8.06 \mathrm{mmol})$ was added. The mixture was vigorously stirred for $1 \mathrm{~h}$, whereupon it was cooled to $0{ }^{\circ} \mathrm{C}$. Aqueous $49 \% \mathrm{HF}$ $(13 \mathrm{~mL}, 364 \mathrm{mmol})$ was added dropwise and the mixture was stirred at $0{ }^{\circ} \mathrm{C}$ for $30 \mathrm{~min}$. After dilution with ether $(100 \mathrm{~mL})$, solid $\mathrm{NaHCO}_{3}(30 \mathrm{~g})$ was added portionwise over $5 \mathrm{~min}$. After stirring for another $5 \mathrm{~min}$, the mixture was filtered through a sintered funnel packed with $\mathrm{MgSO}_{4}$. The solids were well rinsed with ether and the combined filtrate was concentrated in vacuo. The residue was purified by silica gel flash chromatography (petroleum ether-ethyl acetate, 7:3 to 3:2) to afford the lactone $3(0.78 \mathrm{~g}, 3.71 \mathrm{mmol}, 69 \%)$ as a yellow oil and a 1.6:1 mixture of separable diastereomers (found: $M^{+}, 210.1254 . \mathrm{C}_{12} \mathrm{H}_{18} \mathrm{O}_{3}$ requires $M$ 210.1256, 0.7 ppm, EIMS);

3a: $[\alpha]_{\mathrm{D}}^{26}+20.8\left(c 1.73, \mathrm{CHCl}_{3}\right) ; \mathrm{R}_{\mathrm{f}} 0.19$ (petroleum ether-ethyl acetate, 7:3); $v_{\max } / \mathrm{cm}^{-1} 3417,2924$, $1827,1642,1412,1278,1127,914,867 ; \delta_{\mathrm{H}}\left(400 \mathrm{MHz}, \mathrm{CDCl}_{3}\right) 1.22$ (3 H, d, J 7.0), 2.14-2.35 (4 H, m), 2.50 (1 H, br. quin, J 7.0), 3.15 (1 H, dd, J 16.5, 4.5), 3.45 (1 H, dd, J 16.5, 6.5), 3.77-3.83 (1 H, m), 4.45 (1 H, ddd, J 8.5, 6.5, 4.5), $4.94(1 \mathrm{H}, \mathrm{s}), 5.04(1 \mathrm{H}, \mathrm{s}), 5.16$ (1 H, d, J 18.0), 5.17 (1 H, d, J 
11.0), 5.33 (1 H, dddd, $J 18.0,11.0,7.5,7.0) ; \delta_{\mathrm{C}}\left(100 \mathrm{MHz}, \mathrm{CDCl}_{3}\right)$ 16.1, 41.6, 41.8, 43.1, 43.7, 68.9, 73.8, 113.9, 118.6, 134.2, 146.5, 168.1 .

3b: $[\alpha]_{\mathrm{D}}{ }^{26}-14.4\left(c 1.4, \mathrm{CHCl}_{3}\right) ; \mathrm{R}_{\mathrm{f}} 0.13$ (petroleum ether-ethyl acetate, $7: 3$ ); $\mathrm{v}_{\max } / \mathrm{cm}^{-1} 3417,2933$, $1827,1642,1412,1278,1127,913,869 ; \delta_{\mathrm{H}}\left(500 \mathrm{MHz}, \mathrm{CDCl}_{3}\right) 1.10(3 \mathrm{H}, \mathrm{d}, J$ 7.0), 2.16-2.26 (3 H, m), 2.29-2.34 (2 H, m), 2.52-2.58 (1 H, m), 3.13 (1 H, dd, J 16.5, 4.5), 3.48 (1 H, dd, J 16.5, 6.0), 3.78-3.83 (1 H, m), 4.46 (1 H, ddd, J 8.0, 6.0, 4.5), 5.05 (1 H, s), 5.06 (1 H, s), 5.12-5.17 (2 H, m), 5.80-5.89 (1 H, m); $\delta_{\mathrm{C}}\left(125 \mathrm{MHz}, \mathrm{CDCl}_{3}\right)$ 14.7, 41.4, 41.6, 43.0, 43.1, 68.6, 73.4, 114.0, 118.2, 134.4, 146.3, 167.8 .

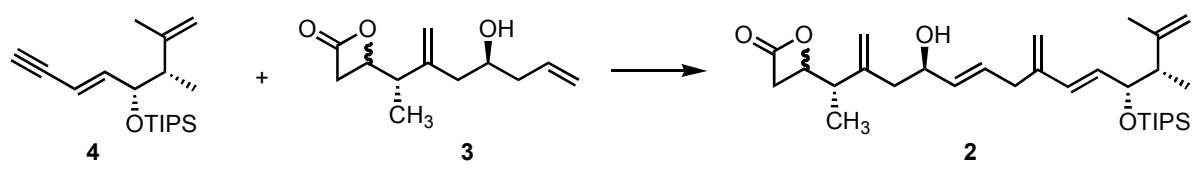

To a solution of alkyne $4(42 \mathrm{mg}, 0.137 \mathrm{mmol})$ and alkene $3(100 \mathrm{mg}, 0.475 \mathrm{mmol})$ in dry acetone $(2.5 \mathrm{~mL})$ at $0{ }^{\circ} \mathrm{C}$ was added $\left[\mathrm{CpRu}\left(\mathrm{CH}_{3} \mathrm{CN}\right)_{3}\right] \mathrm{PF}_{6}(6.0 \mathrm{mg}, 0.0138 \mathrm{mmol})$. The mixture was warmed to r.t. and stirred for $13 \mathrm{~h}$, whereupon it was concentrated in vacuo. The residue was purified by silica gel flash chromatography (petroleum ether-ethyl acetate, 20 to $40 \%$ ) to afford the lactone 2 (52 mg, $0.100 \mathrm{mmol}, 75 \%$ ) as a yellow oil and a 1.6:1 mixture of $C-3$ epimers (Found: $[M+N a]^{+}, 539.3517$. $\mathrm{C}_{31} \mathrm{H}_{52} \mathrm{O}_{4} \mathrm{NaSi}$ requires $\left.M+\mathrm{Na} 539.3533,2.9 \mathrm{ppm}, \mathrm{ESIMS}\right) ;[\alpha]_{\mathrm{D}}{ }^{26}-0.2\left(c 0.85, \mathrm{CHCl}_{3}\right) ; \mathrm{R}_{\mathrm{f}} 0.40$ (petroleum ether-ethyl acetate, 7:3); $v_{\max } / \mathrm{cm}^{-1} 3441,2943,2866,1831,1645,1462,1374,1125,1059$, 970, 882; $\delta_{\mathrm{H}}\left(500 \mathrm{MHz}, \mathrm{CDCl}_{3}\right.$, minor diastereomer in brackets) 0.97 (3 H, d, J 7.0), $1.05(21 \mathrm{H}, \mathrm{s})$, 1.20 (1.09) (3 H, d, J 7.0), 1.75 (3 H, s), 2.18-2.33 (2 H, m), 2.38 (1 H, br quin., J 7.0), 2.90 (2 H, d, J 6.5), 3.12 (3.13) (1 H, dd, J 16.5, 4.5), 3.42 (3.45) (1 H, dd, J 16.5, 5.5), 4.20-4.25 (1 H, m), 4.35-4.38 (1 H, m), 4.43 (4.46) (1 H, ddd, J 7.0, 5.5, 4.5), $4.69(1 \mathrm{H}, \mathrm{s}), 4.78(1 \mathrm{H}, \mathrm{s}), 4.91(2 \mathrm{H}, \mathrm{s}), 4.98$ (5.03) $(1 \mathrm{H}, \mathrm{s}), 5.02$ (5.06) (1 H, s), 5.53 (5.55) (1 H, dd, J 15.0, 7.0), 5.61 (5.62) (1 H, dd, J 16.0, 6.5), 5.76 (5.76) (1 H, dt, J 15.0, 7.0), $6.16(1 \mathrm{H}, \mathrm{d}, J 16.0) ; \delta_{\mathrm{C}}\left(125 \mathrm{MHz}, \mathrm{CDCl}_{3}\right.$, minor diastereomer in brackets) 12.4, 13.2 (13.3), 16.0, 18.1, 21.8, 35.0, 41.6 (41.2), 43.4 (42.9), 43.8 (43.6), 47.7, 71.1 (70.6), 73.8 (73.3), 75.3 (75.4), 111.4, 113.9 (114.1), 115.7, 129.6 (129.3), 130.5 (130.4), 131.8 (131.9), 133.7 (133.6), 143.7 (143.8), 146.0 (145.7), 146.9, 168.1 (167.8).

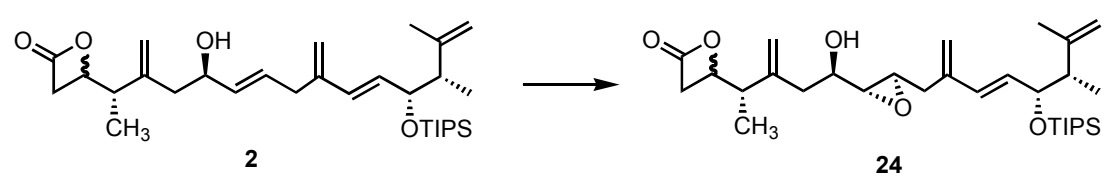

A dry flask was charged with freshly activated powdered $4 \AA$ molecular sieves $(1.5 \mathrm{~g})$ and $\mathrm{CH}_{2} \mathrm{Cl}_{2}(10$ $\mathrm{mL}$ ) and cooled to $-20{ }^{\circ} \mathrm{C}$ (tetrachloroethylene/dry ice). (-)-Diethyl tartrate (102 $\left.\mathrm{mg}, 0.495 \mathrm{mmol}\right)$ in S- 11 - 
$\mathrm{CH}_{2} \mathrm{Cl}_{2}(1 \mathrm{~mL})$ was added, followed with $\mathrm{Ti}(\mathrm{O} i \mathrm{Pr})_{4}(117 \mathrm{mg}, 0.413 \mathrm{mmol})$ in $\mathrm{CH}_{2} \mathrm{Cl}_{2}(1 \mathrm{~mL})$. The mixture was stirred for $30 \mathrm{~min}$, whereupon tert-butylhydroperoxide $(6.2 \mathrm{M}$ in decane, $0.13 \mathrm{~mL}$, $0.806 \mathrm{mmol})$ in $\mathrm{CH}_{2} \mathrm{Cl}_{2}(3 \mathrm{~mL}$, the solution was dried for $30 \mathrm{~min}$ over freshly activated powdered $4 \AA$ molecular sieves) was added. After another $30 \mathrm{~min}$, a solution of alkene 2 (213 mg, $0.412 \mathrm{mmol})$ in $\mathrm{CH}_{2} \mathrm{Cl}_{2}(3 \mathrm{~mL})$ was added. The mixture was stirred at $-20{ }^{\circ} \mathrm{C}$ for $2 \mathrm{~h}$, whereupon citric acid $(0.036$ $\mathrm{M}$ in ether-acetone $10 \% \mathrm{v} / \mathrm{v}, 12.0 \mathrm{~mL}, 0.432 \mathrm{mmol}$ ) was added and the cooling bath was removed. Upon reaching r.t. the mixture was filtered through celite and concentrated in vacuo. The residue was purified by silica gel flash chromatography (petroleum ether-ethyl acetate, 17:3 to 4:1) to afford the C-3 epimer epoxide $24 \mathrm{a}(45 \mathrm{mg}, 0.084 \mathrm{mmol}, 20 \%$ ) as a pale yellow oil and a 1:1 mixture of 24a and the C-3 epimer epoxide 24b (138 mg, $0.259 \mathrm{mmol}, 63 \%$ ) (Found: $[\mathrm{M}+\mathrm{Na}]^{+}, 555.3459$. $\mathrm{C}_{31} \mathrm{H}_{52} \mathrm{O}_{5} \mathrm{NaSi}$ requires $M+N a 555.3482,4.1$ ppm, ESIMS);

24a: $[\alpha]_{\mathrm{D}}^{25}+8.7\left(c 1.26, \mathrm{CHCl}_{3}\right) ; \mathrm{R}_{\mathrm{f}} 0.33$ (petroleum ether-ethyl acetate, $7: 3$ ); $v_{\max } / \mathrm{cm}^{-1} 3473,2943$, 2867, 1830, 1644, 1462, 1374, 1125, 1060, 969, 883; $\delta_{\mathrm{H}}\left(500 \mathrm{MHz}, \mathrm{CDCl}_{3}\right) 0.98$ (3 H, d, J 7.0), 1.05 $(21 \mathrm{H}, \mathrm{s}), 1.20$ (3 H, d, J 7.0), $1.76(3 \mathrm{H}, \mathrm{s}), 2.22$ (1 H, dd, J 15.0, 9.0), 2.33 (1 H, dd, J 15.0, 3.0), 2.36 (1 H, dd, $J 15.0,6.0), 2.40$ (1 H, br. quin, $J$ 6.5), 2.48 (1 H, dd, $J 15.0,6.0), 2.51$ (1 H, br. quin, $J$ 7.5), 2.81 (1 H, dd, J 3.7, 2.2), 3.17 (1 H, td, J 6.0, 2.2), 3.17 (1 H, dd, J 16.5, 4.5), 3.44 (1 H, dd, $J$ 16.5, 5.5), 3.84 (1 H, br. dt, J 9.0, 3.7), 4.40 (1 H, td, J 6.0, 1.0), 4.46 (1 H, ddd, J 8.2, 5.5, 4.5), 4.69 (1 H, br. s), 4.79 (1 H, br. s), $4.92(1 \mathrm{H}, \mathrm{s}), 5.05$ (2 H, s), 5.07 (1 H, s), 5.63 (1 H, dd, J 16.5, 6.5), $6.21\left(1 \mathrm{H}, \mathrm{d}, J\right.$ 16.5); $\delta_{\mathrm{C}}\left(125 \mathrm{MHz}, \mathrm{CDCl}_{3}\right)$ 12.4, 13.0, 16.0, 18.1, 21.9, 34.3, 39.8, 41.6, 43.6, 47.6, 54.0, 60.5, 68.1, 73.7, 75.1, 111.5, 113.8, 116.5, 130.7, 131.8, 141.2, 145.7, 146.7, 168.1.

24b: $R_{f} 0.25$ (petroleum ether-ethyl acetate, 7:3); the NMR data for $24 b$ was deduced from the spectrum of $24 \mathrm{a}$ and that of the mixture $\delta_{\mathrm{H}}\left(500 \mathrm{MHz}, \mathrm{CDCl}_{3}\right) 0.98(3 \mathrm{H}, \mathrm{d}, J 7.0), 1.05(21 \mathrm{H}, \mathrm{s})$, 1.10 (3 H, d, J 7.0), 1.76 (3 H, s), 2.25 (1 H, dd, J 15.0, 9.0), 2.33 (1 H, dd, J 15.0, 3.0), 2.36 (1 H, dd, J 15.0, 6.0), 2.40 (1 H, br. quin, J 6.5), 2.47 (1 H, dd, J 15.0, 6.0), 2.57 (1 H, br. quin, J 7.5), 2.83 (1 H, dd, J 3.7, 2.2), 3.17 (1 H, td, J 6.0, 2.2), 3.13 (1 H, dd, $J 16.5,4.5), 3.47$ (1 H, dd, $J 16.5,5.5)$, 3.86 (1 H, br. dt, J 9.0, 3.7), 4.39 (1 H, td, J 6.0, 1.0), 4.47 (1 H, ddd, J 8.2, 5.5, 4.5), 4.69 (1 H, br. s), 4.79 (1 H, br. s), $4.92(1 \mathrm{H}, \mathrm{s}), 5.04(1 \mathrm{H}, \mathrm{s}), 5.06(1 \mathrm{H}, \mathrm{s}), 5.09(1 \mathrm{H}, \mathrm{s}), 5.63(1 \mathrm{H}, \mathrm{dd}, J$ 16.5, 6.5), $6.21(1 \mathrm{H}, \mathrm{d}, J 16.5) ; \delta_{\mathrm{C}}\left(125 \mathrm{MHz}, \mathrm{CDCl}_{3}\right)$ 12.4, 13.1, 16.0, 18.1, 21.9, 34.4, 39.8, 41.4, 42.9, 47.6, $54.1,60.5,67.6,73.3,75.1,111.5,114.1,116.4,130.6,131.9,141.3,145.3,146.7,167.7$. 


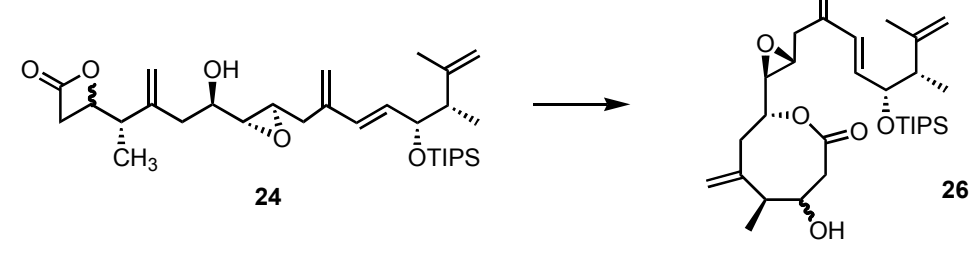

Lactone 24 (1:1 mixture of C-3 epimers, $128 \mathrm{mg}, 0.240 \mathrm{mmol}$ ) and distannoxane 25 (14 mg, 0.011 mmol) were placed in a dry flask, and dry hexane $(120 \mathrm{~mL})$ was added. The mixture was stirred at reflux for $1 \mathrm{~h}$, cooled down and concentrated in vacuo. The residue was purified by silica gel flash chromatography (petroleum ether-ethyl acetate, 17:3) to afford the lactone 26 (119 mg, $0.223 \mathrm{mmol}$, $93 \%)$ as a pale yellow oil and an inseparable $1: 1$ mixture of $C-3$ epimers; $[\alpha]_{\mathrm{D}}{ }^{25}+26.0(c 1.93$, $\mathrm{CHCl}_{3}$ ); $\mathrm{R}_{\mathrm{f}} 0.21$ (petroleum ether-ethyl acetate, 7:3); $v_{\max } / \mathrm{cm}^{-1} 3448,2943,2886,1732,1644,1462$, $1373,1251,1162,1127,1102,1059,1014,992,987,884$

one $\boldsymbol{C}$-3 epimer: $\delta_{\mathrm{H}}\left(500 \mathrm{MHz}, \mathrm{CDCl}_{3}\right) 0.98(3 \mathrm{H}, \mathrm{d}, J$ 7.0), $1.05(21 \mathrm{H}, \mathrm{s}), 1.18(3 \mathrm{H}, \mathrm{d}, J$ J.0), 1.76 (3 H, s), 2.10 (1 H, dq, J 9.5, 7.0), 2.37-2.54 (4 H, m), 2.52 (1 H, dd, J 11.5, 7.0), 2.55 (1 H, dd, $J$ 13.5, 3.0), 2.71 (1 H, dd, J 11.5, 5.0), 2.89 (1 H, dd, J 5.5, 2.5), 3.11 (1 H, td, J 5.5, 2.0), $3.66(1 \mathrm{H}$, m), 4.25 (1 H, ddd, J 11.0, 5.5, 2.0), 4.40 (1 H, br. t, J 6.0), 4.68 (1 H, br. s), 4.78 (1 H, br. s), 5.03 (1 H, br. s), 5.06 (1 H, br. s), 5.07 (1 H, br. s), 5.12 (1 H, br. s), 5.60 (1 H, dd, J 16.0, 6.5), 6.20 (1 H, d, $J$ 16.0); $\delta_{\mathrm{C}}\left(125 \mathrm{MHz}, \mathrm{CDCl}_{3}\right) 12.4,13.0,18.1,21.9,34.17,37.9,41.2,42.7,45.0,47.6,56.4,58.4$, 73.3, 75.1, 81.1, 111.5, 116.7, 118.9, 130.64, 131.8, 140.8, 144.9, 146.8, 171.7.

other $\boldsymbol{C}$-3 epimer: $\delta_{\mathrm{H}}\left(500 \mathrm{MHz} \mathrm{CDCl}_{3}\right) 0.98(3 \mathrm{H}, \mathrm{d}, J$ 7.0), $1.05(21 \mathrm{H}, \mathrm{s}), 1.23(3 \mathrm{H}, \mathrm{d}, J$ 7.0), 1.76 (3 H, s), 2.01 (1 H, dq, J 9.5, 7.0), 2.37-2.54 (4 H, m), 2.49 (1 H, dd, J 12.5, 5.0), 2.61 (1 H, dd, J 14.0, 1.5), 2.91 (1 H, dd, J 5.0, 2.0), 2.97 (1 H, dd, J 12.5, 4.0), 3.14 (1 H, ddd, J 6.0, 5.0, 2.0), 4.10 (1 H, m), 4.40 (1 H, br. t, J 6.0), 4.59 (1 H, ddd, J 11.0, 5.0, 2.5), 4.68 (1 H, br. s), 4.78 (1 H, br. s), 5.07 (2 H, br. s), 5.12 (1 H, br. s), 5.21 (1 H, br. s), 5.60 (1 H, dd, J 16.0, 6.5), 6.20 (1 H, d, J 16.0); $\delta_{\mathrm{C}}$ (125 MHz, $\left.\mathrm{CDCl}_{3}\right)$ 12.4, 13.0, 18.1, 21.9, 34.21, 37.9, 41.2, 42.8, 43.7, 47.6, 55.8, 58.5, 73.3, 75.1, 79.3, 111.5, 116.1, 118.9, 130.68, 131.8, 140.8, 144.9, 147.4, 172.2.
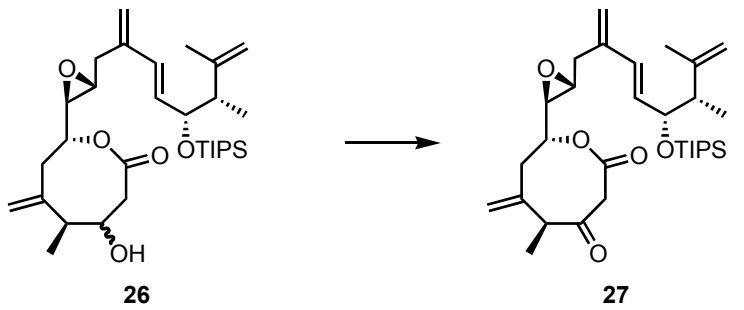

To a solution of alcohol $26(86 \mathrm{mg}, 0.161 \mathrm{mmol})$ in $\mathrm{CH}_{2} \mathrm{Cl}_{2}(2 \mathrm{~mL})$ was added (1,1,1-triacetoxy)-1,1dihydro-1,2-benziodoxol-3(1H)-one $(102 \mathrm{mg}, 0.240 \mathrm{mmol})$. The mixture was stirred for $1 \mathrm{~h}$, 
whereupon $\mathrm{CH}_{2} \mathrm{Cl}_{2}(2 \mathrm{~mL})$ and (1,1,1-triacetoxy)-1,1-dihydro-1,2-benziodoxol-3(1H)-one (102 mg, $0.240 \mathrm{mmol})$ were added. After another $15 \mathrm{~min}$, ether $(50 \mathrm{~mL})$ was added and the mixture was washed with a $1: 1 \mathrm{v} / \mathrm{v}$ solution of saturated aqueous $\mathrm{NaHCO}_{3} / 1 \mathrm{M}$ aqueous $\mathrm{Na}_{2} \mathrm{~S}_{2} \mathrm{O}_{3}(25 \mathrm{~mL})$, water $(25 \mathrm{~mL})$, brine $(25 \mathrm{~mL})$ and dried over $\mathrm{MgSO}_{4}$. The volatiles were removed in vacuo to give a residue that was purified by silica gel flash chromatography (petroleum ether-ether, 9:1) to afford the ketone 27 (70 mg, $0.132 \mathrm{mmol}, 82 \%$ ) as a pale yellow oil (Found: $M+, 530.3420 . \mathrm{C}_{31} \mathrm{H}_{50} \mathrm{O}_{5} \mathrm{Si}$ requires $M 530.3427,1.4 \mathrm{ppm}, \mathrm{EIMS}) ;[\alpha]_{\mathrm{D}}^{23}+31.7$ (c 1.33, $\left.\mathrm{CHCl}_{3}\right) ; \mathrm{R}_{\mathrm{f}} 0.27$ (petroleum ether-ether, $4: 1) ; v_{\max } / \mathrm{cm}^{-1} 2943,2867,1756,1715,1645,1463,1374,1301,1229,1175,1059,1014,989,884 ; \delta_{\mathrm{H}}$ (500 MHz, $\left.\mathrm{CDCl}_{3}\right) 0.99$ (3 H, d, J 7.0), 1.05 (21 H, s), 1.19 (3 H, d, J 7.0), 1.76 (3 H, s), 2.39-2.47 (4 H, m), 2.52 (1 H, dd, J 16.5, 5.5), 3.04 (1 H, dd, J 4.0, 2.0), 3.08 (1 H, td, J 5.5, 2.0), 3.34 (1 H, d, J 13.5), 3.51 (1 H, q, J 7.0), 3.56 (1 H, d, J 13.5), 4.42 (1 H, br. t, J 6.0), 4.69 (1 H, br. s), 4.79 (1 H, br. s), $5.04(1 \mathrm{H}, \mathrm{s}), 5.06(1 \mathrm{H}, \mathrm{s}), 5.09(1 \mathrm{H}, \mathrm{s}), 5.10(1 \mathrm{H}, \mathrm{s}), 5.08-5.12(1 \mathrm{H}$, masked), 5.63 (1 H, dd, 16.0, 6.5), 6.21 (1 H, d, J 16.0); $\delta_{\mathrm{C}}\left(125 \mathrm{MHz}, \mathrm{CDCl}_{3}\right)$ 12.4, 13.0, 16.8, 18.1, 21.9, 34.2, 38.7, 47.6, 52.1, 52.4, 54.9, 57.9, 75.0, 78.7, 111.5, 116.9, 120.6, 130.7, 131.8, 140.8, 143.6, 146.8, 168.2 200.2.

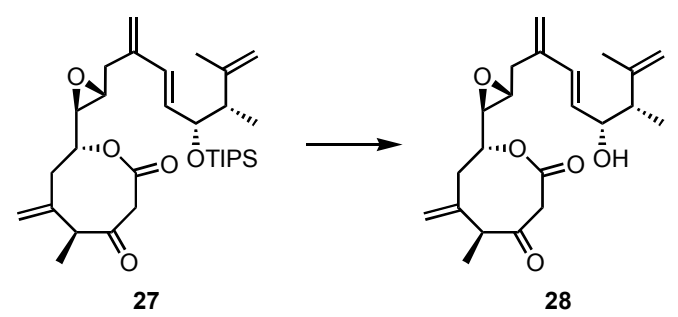

To a solution of lactone $27(33 \mathrm{mg}, 0.062 \mathrm{mmol})$ in THF $(8 \mathrm{~mL})$ at $0{ }^{\circ} \mathrm{C}$ was added TBAF $(1.0 \mathrm{M}$ in THF, $0.3 \mathrm{~mL}, 0.3 \mathrm{mmol}$ ) and the cooling bath was removed. The solution was stirred for $1 \mathrm{~h}$, whereupon it was diluted with ether, washed with water, brine and dried over $\mathrm{MgSO}_{4}$. The volatiles were removed in vacuo to give a residue that was purified by silica gel flash chromatography (petroleum ether-ethyl acetate, 3:1) to afford the alcohol 28 (22 $\mathrm{mg}, 0.059 \mathrm{mmol}, 95 \%)$ as a colorless oil (Found: $[M+N a]^{+}$, 397.1982. $\mathrm{C}_{22} \mathrm{H}_{30} \mathrm{O}_{5} \mathrm{Na}$ requires $M+\mathrm{Na}$ 397.1982, 2.3 ppm, ESIMS); $[\alpha]_{\mathrm{D}}^{25}+46.2\left(c\right.$ 2.08, $\mathrm{CHCl}_{3}$ ); $\mathrm{R}_{\mathrm{f}} 0.20$ (petroleum ether-ethyl acetate, 7:3); $\mathrm{v}_{\max } / \mathrm{cm}^{-1} 3446,2964$, 2931, 1751, 1711, 1645, 1456, 1232, 1176, 1045, 1021, 986, 894; $\delta_{\mathrm{H}}\left(500 \mathrm{MHz}, \mathrm{CDCl}_{3}\right) 0.99(3 \mathrm{H}, \mathrm{d}$, J 7.0), 1.19 (3 H, d, J 7.0), 1.73 (3 H, s), 2.27 (1 H, dq, J 9.0, 7.0), 2.45 (2 H, d, J 8.0), 2.48 (1 H, dd, J 15.5, 5.5), 2.54 (1 H, dd, J 15.5, 5.5), 3.05 (1 H, dd, J 4.5, 2.0), 3.09 (1 H, td, J 5.5, 2.0), 3.35 (1 H, d, J 13.5), 3.51 (1 H, q, J 7.0), 3.56 (1 H, d, J 13.5), 3.95 (1 H, br. t, J 8.0), 4.88 (1 H, br. s), 4.93 (1 H, br. s), $5.04(1 \mathrm{H}, \mathrm{s}), 5.07(1 \mathrm{H}, \mathrm{dt}, J$ 8.0, 4.5), $5.11(1 \mathrm{H}, \mathrm{s}), 5.12(1 \mathrm{H}, \mathrm{s}), 5.16(1 \mathrm{H}, \mathrm{s}), 5.67(1 \mathrm{H}, \mathrm{dd}$, $J$ 16.0, 7.0), 6.35 (1 H, d, J 16.0); $\delta_{\mathrm{C}}\left(125 \mathrm{MHz}, \mathrm{CDCl}_{3}\right)$ 15.6, 16.8, 18.7, 36.2, 39.0, 48.2, 52.1, 52.4, 55.0, 58.0, 74.2, 78.8, 113.7, 117.9, 120.6, 130.6, 133.8, 140.8, 143.5, 146.7, 168.2, 200.2. 


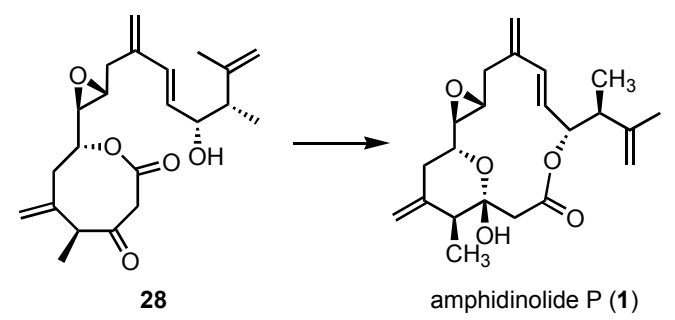

Lactone 28 (14.0 mg, $0.037 \mathrm{mmol})$ and distannoxane $25(9 \mathrm{mg}, 0.007 \mathrm{mmol})$ were placed in a dry flask, and dry hexane $(37 \mathrm{~mL})$ was added. The mixture was stirred at reflux for $8 \mathrm{~h}$, cooled down and concentrated in vacuo. The residue was purified by silica gel flash chromatography (petroleum ether-ether, 17:3) to afford amphidinolide P (1) $(11.7 \mathrm{mg}, 0.031 \mathrm{mmol}, 84 \%)$ as a colorless oil; $[\alpha]_{\mathrm{D}}^{23}-27.4$ (c $\left.0.17, \mathrm{MeOH}\right) ; \mathrm{R}_{\mathrm{f}} 0.35$ (petroleum ether-ethyl acetate, 17:3); $v_{\max } / \mathrm{cm}^{-1} 3482,3084$, 2971, 2942, 1712, 1650, 1433, 1376, 1361, 1291, 1243, 1189, 1111, 988, 967, 896; $\delta_{\mathrm{H}}(500 \mathrm{MHz}$, $\left.\mathrm{C}_{6} \mathrm{D}_{6}\right) 0.91$ (3 H, d, J 7.0), 0.92 (3 H, d, J 7.0), 1.67 (3 H, br. s), 1.93-1.96 (1 H, m), 2.10 (1 H, dd, J 12.7, 11.5), 2.17 (1 H, br. dd, J 13.5, 9.5), 2.27 (1 H, d, J 12.0), 2.36 (1 H, d, J 12.0), 2.43 (1 H, dq, J 9.5, 7.0), 2.48 (1 H, dt, J 9.5, 1.5), 2.52 (1 H, dd, J 12.7, 2.7), 2.62 (1 H, dd, J 8.5, 1.5), 2.68 (1 H, br. d, J 13.5), 3.47 (1 H, ddd, J 11.5, 8.5, 2.7), 4.27 (1 H, d, J 2.0), 4.77 (1 H, m), 4.81 (1 H, br. s), 4.814.82 (1 H, m), 4.87-4.89 (1 H, m), 4.89-4.90 (1 H, m), $4.94(1 \mathrm{H}, \mathrm{m}), 5.29$ (1 H, br. t, J 8.5), 5.60 (1 $\mathrm{H}, \mathrm{dd}, J 16.2,7.5), 6.20$ (1 H, d, J 16.2); $\delta_{\mathrm{C}}\left(125 \mathrm{MHz}, \mathrm{C}_{6} \mathrm{D}_{6}\right) 11.8,16.1,19.5,36.3,39.4,45.0$ (x2), $45.2,58.2,62.7,73.5,78.5,99.2,110.0,112.3,118.2,129.1,133.6,142.2,143.7,146.5,172.4$.

Note: Data for synthetic 1 was identical to the data reported for the natural product, except for the optical rotation: $[\alpha]_{23}^{\mathrm{D}}-27.4(c 0.17, \mathrm{MeOH})$, lit. $[\alpha]_{20}^{\mathrm{D}}+31$ (c 0.098, MeOH) (Ishibashi, M.; Takahashi, J.; Kobayashi, J. J. Org. Chem. 1995, 60, 6062). Four optical rotation measurements in absolute methanol at slightly different concentrations gave consistent values. Concentrations of $0.09,0.17,0.19,0.23$ gave $[\alpha]_{23}^{\mathrm{D}}$ values of - 27.2, - 27.4, - 31.7 and - 28.3, respectively. We did not observe any change of optical rotation after $5 \mathrm{~h}$ of storage in methanol, and the $1 \mathrm{H}$ NMR spectra of 1 in $\mathrm{C}_{6} \mathrm{D}_{6}$ and $\mathrm{CD}_{3} \mathrm{OD}$ were also unchanged.

Williams et al. reported a synthesis of 1 which relied on two Sharpless asymmetric epoxidations to introduce the chirality, both of them using the $(+)$-diethyl tartrate ligand, and which should give synthetic 1 of opposite absolute configuration to the one reported herein. Yet they also reported a negative optical rotation, $[\alpha]_{23}^{\mathrm{D}}-30$ (c 0.09, MeOH) (Williams, D. R.; Myers, B. J.; Mi, L. Org. Lett. 2000, 2, 945). Unfortunately, Professor Williams was not able to provide us with a sample of synthetic 1 , and no direct comparative measurement could be done. 
S- 16 - 


\begin{tabular}{|c|c|c|c|c|c|}
\hline assignment & $\begin{array}{l}\text { natural amphidinolide } \mathrm{P} \\
{ }^{1} \mathrm{H} \text { NMR spectrum } \\
\left(\mathrm{C}_{6} \mathrm{D}_{6}\right)\end{array}$ & $\begin{array}{l}\text { adjusted } \\
\text { chemical } \\
\text { shift* (= } \\
\text { value - } \\
0.04)\end{array}$ & $\begin{array}{l}\text { synthetic } \\
\text { amphidinolide P } \\
{ }^{1} \mathrm{H} \text { NMR }\left(\mathrm{C}_{6} \mathrm{D}_{6}\right)\end{array}$ & $\begin{array}{l}\text { natural } \\
\text { amphidinolide } \mathrm{P} \\
{ }^{13} \mathrm{C} \mathrm{NMR}\left(\mathrm{C}_{6} \mathrm{D}_{6}\right)\end{array}$ & $\begin{array}{l}\text { synthe } \\
\text { amphic } \\
{ }^{13} \mathrm{C} \mathrm{N}\end{array}$ \\
\hline 1 & & & & 172.4 & 172.4 \\
\hline $2 \mathrm{a}$ & $2.41(\mathrm{~d}, 12)$ & 2.37 & $2.36(\mathrm{~d}, 12)$ & 45.0 & 45.0 \\
\hline $2 \mathrm{~b}$ & $2.31(\mathrm{~d}, 12)$ & 2.27 & $2.27(\mathrm{~d}, 12)$ & & \\
\hline 3 & & & & 99.2 & 99.2 \\
\hline OH-3 & $4.31(\mathrm{~d}, 1.5)$ & 4.27 & $4.27(\mathrm{~d}, 2.0)$ & & \\
\hline 4 & 1.99 (br. q, 6.9) & 1.95 & $1.93-1.96(\mathrm{~m})$ & 45.2 & 45.2 \\
\hline 5 & & & & 143.7 & 143.7 \\
\hline $6 a$ & $2.56(\mathrm{dd}, 12.7,2.5)$ & 2.52 & $2.52(\mathrm{dd}, 12.7,2.7)$ & 39.4 & 39.4 \\
\hline $6 b$ & $2.14(\mathrm{dd}, 12.7,11.7)$ & 2.10 & $2.10(\mathrm{dd}, 12.7,11.5)$ & & \\
\hline 7 & 3.51 (ddd, 11.7, 8.3, 2.5) & 3.47 & $3.47(\mathrm{ddd}, 11.5,8.5,2.7)$ & 73.5 & 73.5 \\
\hline 8 & $2.66(\mathrm{dd}, 8.3,1.4)$ & 2.62 & $2.62(\mathrm{dd}, 8.5,1.5)$ & 62.8 & 62.7 \\
\hline 9 & $2.52(\mathrm{dd}, 9.5,1.4)$ & 2.48 & $2.48(\mathrm{dt}, 9.5,1.5)$ & 58.2 & 58.2 \\
\hline $10 \mathrm{a}$ & $2.72(\mathrm{~d}, 13.9)$ & 2.68 & 2.68 (br. d, 13.5) & 36.4 & 36.3 \\
\hline $10 \mathrm{~b}$ & $2.21(\mathrm{dd}, 13.9,9.5)$ & 2.17 & 2.17 (br. dd, 13.5, 9.5) & & \\
\hline 11 & & & & 142.3 & 142.2 \\
\hline 12 & $6.24(\mathrm{~d}, 16.2)$ & 6.20 & $6.20(\mathrm{~d}, 16.2)$ & 133.6 & 133.6 \\
\hline 13 & $5.64(\mathrm{dd}, 16.2,7.5)$ & 5.60 & $5.60(\mathrm{dd}, 16.2,7.5)$ & 129.1 & 129.1 \\
\hline 14 & $5.34(\mathrm{dd}, 9.3,7.5)$ & 5.30 & 5.29 (br. t, 8.5) & 78.5 & 78.5 \\
\hline 15 & $2.47(\mathrm{qd}, 9.3,7.3)$ & 2.43 & $2.43(\mathrm{dq}, 9.5,7.0)$ & 45.0 & 45.0 \\
\hline 16 & & & & 146.5 & 146.5 \\
\hline $17 \mathrm{a}$ & 4.93 (br. s) & 4.89 & $4.89-4.90(\mathrm{~m})$ & 112.3 & 112.3 \\
\hline $17 \mathrm{~b}$ & 4.92 (br. s) & 4.88 & $4.87-4.89(\mathrm{~m})$ & & \\
\hline $18(3 \mathrm{H})$ & $0.96(\mathrm{~d}, 6.9)$ & 0.92 & $0.92(\mathrm{~d}, 7.0)$ & 16.1 & 16.1 \\
\hline $19 \mathrm{a}$ & 4.86 (br. s) & 4.82 & $4.81-4.82(\mathrm{~m})$ & 110.0 & 110.0 \\
\hline $19 b$ & 4.81 (br. s) & 4.77 & $4.77(\mathrm{~m})$ & & \\
\hline $20(3 \mathrm{H})$ & $0.95(\mathrm{~d}, 7.3)$ & 0.91 & $0.91(\mathrm{~d}, 7.0)$ & 11.8 & 11.8 \\
\hline $21(3 \mathrm{H})$ & $1.71(\mathrm{~s})$ & 1.67 & 1.67 (br. s) & 19.5 & 19.5 \\
\hline $22 \mathrm{a}$ & 4.98 (br. s) & 4.94 & $4.94(\mathrm{~m})$ & 118.1 & 118.2 \\
\hline $22 b$ & 4.85 (br. s) & 4.81 & 4.81 (br. s) & & \\
\hline
\end{tabular}

${ }^{*}$ The scale of the ${ }^{1} \mathrm{H}$ NMR spectrum for natural amphidinolide P had the benzene chemical shift off by $0.04 \mathrm{ppm}$ from the accepted value. The values in this column are adjusted to place the benzene peak at its accepted value of $7.16 \mathrm{ppm}$. 


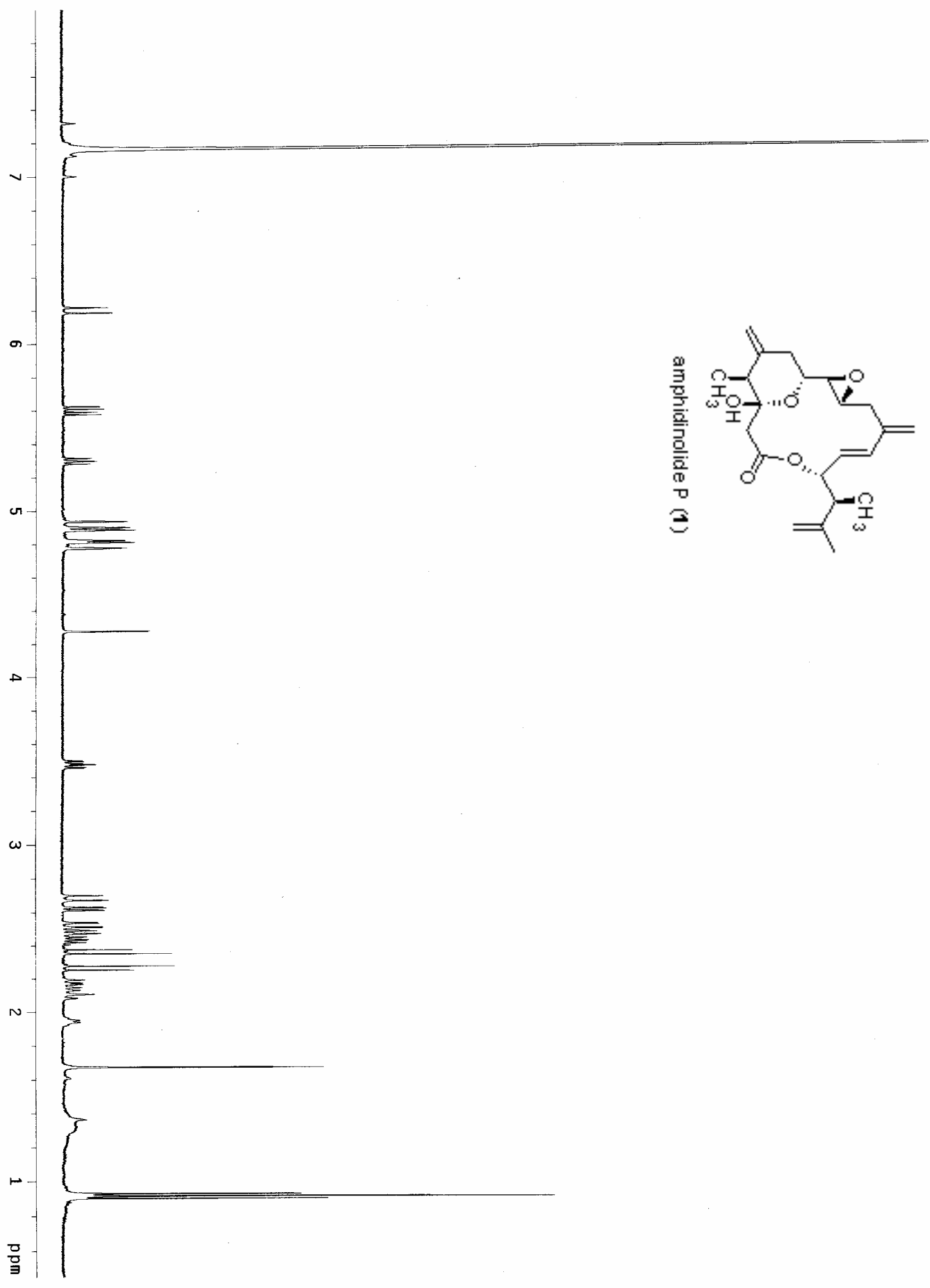




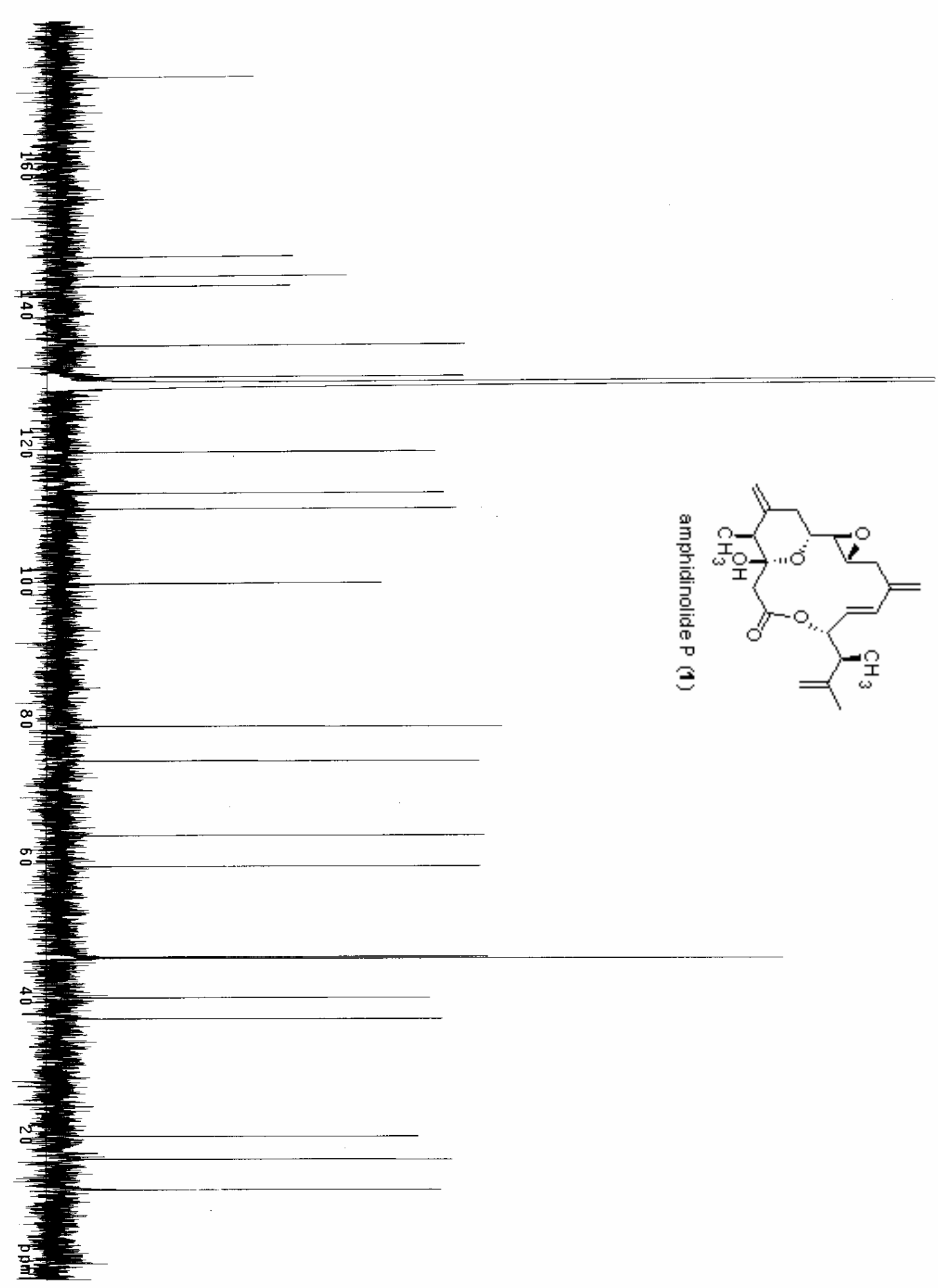

S- 19 - 